\title{
EIGENFUNCTIONS, TRANSFER MATRICES, AND ABSOLUTELY CONTINUOUS SPECTRUM OF ONE-DIMENSIONAL SCHRÖDINGER OPERATORS
}

\author{
Yoram LAST AND BARRY SimON* \\ Division of Physics, Mathematics, and Astronomy \\ California Institute of Technology \\ Pasadena, CA 91125
}

\section{$\S 1$. Introduction}

In this paper, we will primarily discuss one-dimensional discrete Schrödinger operators

$$
(h u)(n)=u(n+1)+u(n-1)+V(n) u(n)
$$

on $\ell^{2}(\mathbb{Z})$ (and the half-line problem, $h_{+}$, on $\left.\ell^{2}(\{n \in \mathbb{Z} \mid n>0\}) \equiv \ell^{2}\left(\mathbb{Z}^{+}\right)\right)$with $u(0)=0$ boundary conditions. We will also discuss the continuum analog

$$
(H u)(x)=-u^{\prime \prime}(x)+V(x) u(x)
$$

on $L^{2}(\mathbb{R})$ (and its half-line problem, $H_{+}$, on $L^{2}(0, \infty)$ with $u(0)=0$ boundary conditions).

We will focus on a new approach to the absolutely continuous spectrum $\sigma_{\mathrm{ac}}(h)$ and, more generally, $\Sigma_{\mathrm{ac}}(h)$, the essential support of the a.c. part of the spectral measures.

What is new in our approach is that it relies on estimates on the transfer matrix, that is, the $2 \times 2$ matrix $T_{E}(n, m)$ which takes $\left(\begin{array}{c}u(m+1) \\ u(m)\end{array}\right)$ to $\left(\begin{array}{c}u(n+1) \\ u(n)\end{array}\right)$ for solutions $u$ of $h u=E u$ (in the continuum case use $\left(\begin{array}{c}u^{\prime}(x) \\ u(x)\end{array}\right)$ instead of $\left.\left(\begin{array}{c}u(x+1) \\ u(x)\end{array}\right)\right)$. We let $T_{E}(n) \equiv T_{E}(n, 0)$. For example, we will prove the following:

* This material is based upon work supported by the National Science Foundation under Grant No. DMS-9401491. The Government has certain rights in this material.

Typeset by $\mathcal{A M}_{\mathcal{M}}-\mathrm{T}_{\mathrm{E}} \mathrm{X}$ 
Theorem 1.1. Let $h_{+}$be the operator (1.1D) on $\ell^{2}(\{n \in \mathbb{Z} \mid n>0\})$ with $u(0)=0$ boundary conditions. Let

$$
S=\left\{E \mid \underset{L \rightarrow \infty}{\lim _{L \rightarrow \infty}} \frac{1}{L} \sum_{n=1}^{L}\left\|T_{E}(n)\right\|^{2}<\infty\right\} .
$$

Then $S$ is an essential support of the a.c. part of the spectral measure for $h_{+}$(i.e., $\left.S=\Sigma_{\mathrm{ac}}(h)\right)$ and $S$ has zero measure with respect to the singular part of the spectral measure.

The behavior of the transfer matrix is a reflection of the behavior of eigenfunctions since $T$ is built out of eigenfunctions. Indeed, if $u$ and $w$ are any two linearly independent solutions of $h u=E u$ normalized at 0 , then $\frac{1}{L} \sum_{n=1}^{L}\|T(n)\|^{2}$ and $\frac{1}{L} \sum_{n=1}^{L+1}\left[|u(n)|^{2}+\right.$ $\left.|w(n)|^{2}\right]$ are comparable and so Theorem 1.1 relates the a.c. spectrum to the behavior of eigenfunctions.

That there is a connection between eigenfunctions and a.c. spectrum is not new. Gilbert-Pearson [15] related a.c. spectrum to subordinate solutions. Typical is the following (actually due to [26]; see also [23,24]): Call a solution $u$ of $h u=E u$ subordinate if and only if for any linearly independent solution $w$,

$$
\sum_{n=1}^{L}|u(n)|^{2} / \sum_{n=1}^{L}|w(n)|^{2} \rightarrow 0
$$

as $L \rightarrow \infty$. Let

$$
S_{0}=\{E \mid \text { there is no subordinate solution }\} .
$$

Then $S_{0}$ is an essential support of the a.c. part of the spectral measure for $h_{+}$and $S_{0}$ has zero measure with respect to the singular part of the spectral measure.

The Gilbert-Pearson theory provides one-half of the proof of Theorem 1.1. Indeed, we will show that $S \subset S_{0}$. The other direction is intimately related to some new eigenfunction estimates which we discuss in Section 2. Its relation to the theory of Browder, Berezinski, Garding, Gel'fand, and Kac is discussed in the appendix.

Related to Theorem 1.1 is the following, which also relies on the eigenfunction estimate of Section 2:

Theorem 1.2. Let $h_{+}$be as in Theorem 1.1. Let $m_{j}, k_{j}$ be arbitrary sequences in $\{n \in \mathbb{Z} \mid n>0\}$ and let

$$
S_{1}=\left\{E \mid \lim _{j \rightarrow \infty}\left\|T_{E}\left(m_{j}, k_{j}\right)\right\|<\infty\right\} .
$$

Then $S_{1}$ supports the a.c. part of the spectral measure for $h_{+}$in that $\rho_{\mathrm{ac}}\left(\mathbb{R} \backslash S_{1}\right)=0$.

These two theorems allow us to recover virtually all the major abstract results proven in the past fifteen years on the a.c. spectrum for ergodic Schrödinger operators with the 
exception of Kotani's results [30,32] on $\{E \mid \gamma(E)=0\}$. More significantly, they establish new results and settle an important open problem. Among the results recovered via a new proof are the Ishii-Pastur theorem [21,38], Kotani's support theorem [31], and the results of Simon-Spencer [46].

In a companion paper with A. Kiselev [29], we will use Theorems 1.1, 1.2 and Theorem 1.3 below to analyze, recover, and extend results on decaying random potentials [44,12,11], sparse potentials [39,40], and $n^{-\alpha}\left(1>\alpha>\frac{3}{4}\right)$ potentials [28].

Theorem 1.1 and Fatou's lemma immediately imply that if $Q$ is any subset of $\mathbb{R}$ and

$$
\sup _{n} \int_{Q}\left\|T_{E}(n)\right\|^{2} d E<\infty
$$

then $Q$ lies in the essential support of $d \rho_{\text {ac }}$ (for Fatou's lemma and (1.3) show for a.e. $E \in$ $Q$ we have that $\left.\underline{\lim } \frac{1}{L} \sum_{n=1}^{L}\left\|T_{E}(n)\right\|^{2}<\infty\right)$ but (1.3) does not seem to eliminate the possibility of singular spectrum on $Q$ (on the set of Lebesgue measure zero where Fatou does not apply). In this regard, the following result, which is an extension of ideas of Carmona [4], is of interest:

Theorem 1.3. Suppose that

$$
\varliminf_{n \rightarrow \infty} \int_{a}^{b}\left\|T_{E}(n)\right\|^{p} d E<\infty
$$

for some $p>2$. Then the spectrum is purely absolutely continuous on $(a, b)$.

It is interesting to compare Theorems 1.2 and 1.3. A priori, one might think there could be potentials so there exist $n_{1}<m_{1}<n_{2}<m_{2}<\cdots$ (with the $m_{j}-n_{j}$ and $n_{j+1}-m_{j}$ growing very rapidly) so that $T_{E}(n)$ is bounded at the $m_{j}$ and unbounded at the $n_{j}$. While this could happen at a single $E$, by these two theorems it cannot happen for all $E$ in $(a, b)$.

To describe our most important new result, we define

Definition. Let $V, W$ be bounded functions on $\{n \in \mathbb{Z} \mid n>0\}$. We say that $W$ is a right limit of $V$ if and only if there exist $n_{j} \rightarrow \infty$ so that $V\left(n+n_{j}\right) \rightarrow W(n)$ as $j \rightarrow \infty$ for each fixed $n>0$.

Then we will prove from Theorem 1.1 and the eigenfuction expansion results of Section 2 that

Theorem 1.4. If $W$ is a right limit of $V$ and $\tilde{h}_{+}, h_{+}$are the half-line Schrödinger operators associated to $W, V$ respectively, then $\Sigma_{\mathrm{ac}}\left(h_{+}\right) \subset \Sigma_{\mathrm{ac}}\left(\tilde{h}_{+}\right)$.

Remark. This result is particularly interesting because it is easy to see that $\sigma_{\text {ess }}\left(\tilde{h}_{+}\right) \subset$ $\sigma_{\text {ess }}\left(h_{+}\right)$with the inclusion in the opposite direction.

Our proof of Theorem 1.4 depends on the shift to transfer matrices rather than eigenfunctions.

This theorem will have an important corollary: 
Theorem 1.5. Let $W$ be an almost periodic function on $\mathbb{Z}$ (resp. $\mathbb{R})$. Let $h$ (resp. $H$ ) be the full-line operator given by (1.1). For each $W_{\omega}$ in the hull of $W$, let $h_{\omega}$ (resp. $\left.H_{\omega}\right)$ be the corresponding operator. Then the a.c. spectrum, indeed the essential support of the a.c. spectrum, of $h_{\omega}$ is independent of $\omega$.

Remarks. 1. The result holds more generally than almost periodic potentials. It suffices that the underlying process be minimally ergodic.

2. We will also recover the Deift-Simon [10] result that the multiplicity of the a.c. spectrum is 2 .

3. Following Pastur [38] and others (see [5,7]), it is known that the spectrum and its components are a.e. constant on the hull. In 1982, Avron-Simon [2] proved that the spectrum is everywhere constant rather than a.e. constant in the almost periodic case. Theorem 1.5 has been believed for a long time, but this is its first proof. It is known (see Jitomirskaya-Simon [25]) that the s.c. and p.p. components need not be everywhere constant.

In this paper, we will also obtain rigorous spectral results on the operator $h_{+}$where $V(n)=\lambda \cos \left(n^{\beta}\right)$, and $1<\beta$ is not an integer.

Theorem 1.5 is reminiscent of the invariance of the a.c. spectrum under rank one perturbations for all couplings. This is no coincidence. In our development of Theorems 1.1-1.2, what distinguishes a.c. spectrum from non-a.c. spectrum is its invariance under boundary conditions.

While the main focus of this paper is on the a.c. spectrum and transfer matrices, we will say something about point spectrum also. In this introduction, we will focus on the discrete case with $V$ bounded. In [47], using constancy of the Wronskian, Simon-Stolz proved

Theorem $1.6([47])$. If $\sum_{n=1}^{\infty}\left\|T_{E}(n)\right\|^{-2}=\infty$, then $h u=$ Eu has no solution which is $L^{2}$ at infinity.

As we will see in Section 8, it can happen that $\sum_{n=1}^{\infty}\left\|T_{E}(n)\right\|^{-2}<\infty$ without there being a solution $L^{2}$ at infinity; indeed, without there even being a bounded solution, but $\sum_{n=1}^{\infty}\left\|T_{E}(n)\right\|^{-2}<\infty$ has one important consequence. Call a solution $u$ of $h u=E u$ strongly subordinate if for any linearly independent solution $w$ we have that

$$
\left[u(n)^{2}+u(n+1)^{2}\right] /\left[w(n)^{2}+w(n+1)^{2}\right] \rightarrow 0
$$

as $n \rightarrow \infty$. It is easy to see that any strongly subordinate solution is subordinate. We will prove that

Theorem 1.7. If $V$ is bounded and $\sum_{n=1}^{\infty}\left\|T_{E}(n)\right\|^{-2}<\infty$, then there is a strongly subordinate solution of $h u=E u$. This solution, $u_{\infty}$, obeys the estimate

$$
\left\|u_{\infty}(n)\right\|^{2} \leq\left\|T_{E}(n)\right\|^{-2}+\frac{\pi^{2}}{4}\left\|T_{E}(n)\right\|^{2}\left(\sum_{m=n}^{\infty} \frac{1}{\left\|T_{E}(m)\right\|^{2}}\right)^{2} .
$$


In particular, if

$$
\sum_{n=1}^{\infty}\left\{\left\|T_{E}(n)\right\|^{2}\left(\sum_{m=n}^{\infty}\left\|T_{E}(m)\right\|^{-2}\right)^{2}\right\}<\infty,
$$

then $h u=E u$ has an $L^{2}$ solution.

Theorem 1.7 is essentially an abstraction of a well-known argument of Ruelle [41]. We will use it in $[29,36]$ to prove point spectrum in certain models, including new and simplified proofs of the results of Simon [44] and some of the results of Gordon [17].

The plan of this paper is as follows. In Section 2 we develop eigenfunction estimates. Their relation to the BGK eigenfunction expansions is discussed in the appendix which includes higher-dimensional results. In Section 3 we use the results of Section 2 and the Gilbert-Pearson theory to prove Theorems 1.1 and 1.2 and we will use Carmona's formula to prove Theorem 1.3. In Section 4 we recover and extend the Simon-Spencer [46] results. In Section 5 we prove Theorem 1.4 and in Section 6 we prove Theorem 1.5 and some other consequences of Theorem 1.4, including the Kotani support theorem. In Section 7 we discuss $\lambda \cos \left(n^{\beta}\right)$. In Section 8 we prove Theorems 1.7 and 1.8.

We would like to thank Bert Hof and Svetlana Jitomirskaya for useful discussions. B.S. would like to thank M. Ben-Artzi for the hospitality of the Hebrew University where some of this work was done.

\section{$\S$ 2. Eigenfunction Estimates}

We consider half-line problems in this section. In the discrete case for fixed $V(n)$ and $z \in \mathbb{C}$, define $u_{D}(n), u_{N}(n)$ to be the solution of $h u=z u$ ( $h$ given by (1.1D)) with boundary conditions

$$
\begin{array}{ll}
u_{D}(0)=0 & u_{D}(1)=1 \\
u_{N}(0)=1 & u_{N}(1)=0 .
\end{array}
$$

We will use $X$ to denote $D$ or $N$ in formulas where either is valid, and $Y$ for the opposite condition.

In the continuum case, $u_{D}, u_{N}$ obey $H u=z u$ ( $H$ given by $\left.(1.1 \mathrm{C})\right)$ with boundary conditions

$$
\begin{array}{ll}
u_{D}(0)=0 & u_{D}^{\prime}(0)=1 \\
u_{N}(0)=1 & u_{N}^{\prime}(0)=0 .
\end{array}
$$

Of course, $u$ is $z$-dependent and we will sometimes use $u(\cdot ; z)$. It is standard that $u(n ; z)$, $u(x ; z)$, and $u^{\prime}(x ; z)$ are entire functions of $z$ for real $x, n$.

The solutions $u$ are related to the transfer matrix by

$$
T_{E}(n)=\left(\begin{array}{cc}
u_{N}(n+1) & u_{D}(n+1) \\
u_{N}(n) & u_{D}(n)
\end{array}\right)
$$


in the discrete case and

$$
T_{E}(x)=\left(\begin{array}{ll}
u_{N}^{\prime}(x) & u_{D}^{\prime}(x) \\
u_{N}(x) & u_{D}(x)
\end{array}\right)
$$

in the continuum case.

For $z \in \mathbb{C}_{+}=\{z \mid \operatorname{Im} z>0\}$, there is a unique solution $L^{2}$ at $+\infty$ (for arbitrary $V$ in the discrete case and for $V$ which is limit point at infinity in the continuum case). Both it and its derivative (in the continuum case) are everywhere non-vanishing. In the continuum, we denote the solution by $\varphi_{+}^{D}(x ; z)$ if normalized by $\varphi_{+}^{D}(0 ; z)=1$ and $\varphi_{+}^{N}(x ; z)$ if normalized by $\left(\varphi_{+}^{N}\right)^{\prime}(0 ; z)=-1$, and in the discrete case $\varphi_{+}^{D}(0 ; z)=1, \varphi_{+}^{N}(1 ; z)=-1$. This normalization is chosen so that the Wronskian of $\varphi_{+}^{X}$ and $u_{X}$ is +1 .

The $m$-functions are defined by

$$
\varphi_{+}^{X}(\cdot ; z)= \pm u_{Y}(\cdot ; z)+m_{X}(z) u_{X}(\cdot ; z)
$$

where we take the plus sign in case $X=D$ and minus in case $X=N$. (Noting that the Wronskian of $\varphi_{+}^{X}$ and $\varphi_{+}^{Y}$ is zero, we see that $m_{X}(z) m_{Y}(z)=-1$.)

It is well known (see, e.g., $[5,27]$ ) that the $m$-functions are Herglotz (i.e., analytic with $\operatorname{Im} m>0$ on $\left.\mathbb{C}_{+}\right)$and that the measures

$$
d \rho^{X}(E)=\lim _{\epsilon \downarrow 0} \frac{1}{\pi} \operatorname{Im} m_{X}(E+i \epsilon) d E
$$

are spectral measures for the operator $H_{X}$ ( $h$ or $H$ with appropriate boundary conditions; i.e., in the continuum case $H_{D, N}$ are defined on $L^{2}(0, \infty)$ with $u(0)$ or $u^{\prime}(0)$ boundary conditions, and in the discrete case $H_{D}\left(\right.$ resp. $\left.H_{N}\right)$ is defined on $\ell^{2}\left(\mathbb{Z}_{+}\right)\left(\right.$resp. $\left.\ell^{2}(\{2,3, \ldots\}),\right)$ with $u(0)=0$ (resp. $u(1)=0$ ) boundary conditions). That is, $H_{X}$ is unitarily equivalent to multiplication by $E$ on $L^{2}\left(\mathbb{R}, d \rho^{X}(E)\right.$ ). Note that in (2.3) (and similarly (2.8) below), the limit is intended in the weak sense, that is, holds when smeared in $E$ with continuous functions of compact support.

In the discrete case and in the continuum case with $X=N$, we have

$$
\int \frac{d \rho^{X}(E)}{|E|+1}<\infty
$$

and

$$
m_{X}(z)=\int \frac{d \rho^{X}(E)}{E-z} .
$$

In the continuum case with $X=D$, we only have

$$
\int \frac{d \rho^{D}(E)}{E^{2}+1}<\infty
$$

and a Herglotz representation

$$
m_{D}(z)=a_{0}+\int\left(\frac{1}{E-z}-\frac{E}{1+E^{2}}\right) d \rho^{D}(E)
$$

for a suitable real constant $a_{0}$.

We are heading toward a proof of the following theorems: 
Theorem 2.1D. In the discrete case, for any $V$ and $n$,

$$
\int\left|u_{X}(n ; E)\right|^{2} d \rho^{X}(E)=1
$$

Theorem 2.1C. In the continuum case for any $V \geq 0$ and all $x$,

$$
\int \frac{\left|u_{X}(x ; E)\right|^{2}}{E+1} d \rho^{X}(E) \leq \frac{1}{2}\left(1 \mp e^{-2|x|}\right)
$$

where $\mp$ correspond to $X=D / N$. Moreover, for a universal constant $C$, we have that for all $x$

$$
\int \frac{\left[\int_{x-1}^{x+1}\left|u_{X}^{\prime}(y ; E)\right|^{2} d y\right]}{(E+1)^{2}} d \rho^{X}(E) \leq C .
$$

Remarks. 1. In $(2.6 \mathrm{C}(\mathrm{b}))$, if $x<1$, interpret $x-1$ as 0 .

2. Obviously, $V \geq 0$ can be replaced by $V \geq c$ for any $c$ if $(E+1)^{-1}$ in $(2.6 \mathrm{C})$ is replaced by $(E-c+1)^{-1}$. The proof shows that as long as $-V \leq \alpha(-\Delta)+\beta$ for some $\alpha<1$, estimates similar to $\left(2.6 \mathrm{C}\right.$ ) hold (with $(E+1)^{-1}$ replaced by $\left(E+|\beta|+(1-\alpha)^{-1}\right)$ and the $\frac{1}{2}$ (resp. 1$)$ in the inequality replaced by $\frac{1}{2}(1-\alpha)^{-1}\left(\operatorname{resp} .(1-\alpha)^{-1}\right)$. Thus, the result allows any $V$ whose negative part is uniformly locally $L^{1}$.

As a preliminary we note that

Lemma 2.2. (a) $\epsilon^{2}|m(E+i \epsilon)| \rightarrow 0$ as $\epsilon \downarrow 0$ uniformly for $E$ in compact subsets of $\mathbb{R}$.

(b) $\epsilon|\operatorname{Re} m(E+i \epsilon)| \rightarrow 0$ as $\epsilon \downarrow 0$ and is uniformly bounded for $E$ in compacts.

Proof. (a) is a direct consequence of $(2.4 / 2.5)$. (b) follows from those formulas and the dominated convergence theorem.

The resolvent, $\left(H_{X}-z\right)^{-1}$, of the operator $H_{X}$ has a continuous integral kernel (in the continuum case). In general, this kernel $G_{X}(x, y ; z)$ has the form

$$
G_{X}(x, y ; z)=u_{X}\left(x_{<} ; z\right) \varphi_{+}^{X}\left(x_{>} ; z\right)
$$

where $x_{<}=\min (x, y), x_{>}=\max (x, y)$. This formula is easy to verify and shows that $G$ is continuous.

\section{Theorem 2.3.}

$$
\lim _{\epsilon \downarrow 0} \frac{1}{\pi} \operatorname{Im} G_{X}(x, x ; E+i \epsilon) d E=\left|u_{X}(x, E)\right|^{2} d \rho^{X}(E) .
$$

Proof. By (2.7) and (2.2),

$$
G_{X}(x, x ; E+i \epsilon)= \pm u_{Y}(x, E+i \epsilon) u_{X}(x ; E+i \epsilon)+m_{X}(E+i \epsilon) u_{X}(x ; E+i \epsilon)^{2} .
$$


Since $u_{X, Y}$ are entire and real for $z$ real, we have that $\lim _{\epsilon \downarrow 0} \operatorname{Im} u_{Y}(x, E+i \epsilon) u_{X}(x, E+$ $i \epsilon)=0$. Similarly, $u_{X}(x ; E+i \epsilon)^{2}=u_{X}(x ; E)^{2}+i \epsilon a(x ; E)+O\left(\epsilon^{2}\right)$ where $u_{X}^{2}$ and $a(x)$ are real. Thus,

$$
\operatorname{Im}\left[m_{X}(E+i \epsilon) u_{X}(x ; E+i \epsilon)^{2}\right]=1+2+3
$$

with

by (2.3) and

$$
1=u_{X}(x ; E)^{2} \operatorname{Im} m_{X}(E+i \epsilon) \rightarrow \pi\left|u_{X}(x, E)\right|^{2} d \rho^{X}(E)
$$

$$
2=\epsilon a(x ; E) \operatorname{Re} m(E+i \epsilon) \rightarrow 0
$$

by Lemma $2.2(\mathrm{~b})$ and

$$
3=\operatorname{Im}\left[O\left(\epsilon^{2}\right) m(E+i \epsilon)\right] \rightarrow 0
$$

by Lemma 2.2(a). Thus, (2.8) is proven.

Remarks. 1. (2.8) is essentially a version of the spectral theorem. We will discuss this further in the appendix.

2. The same method shows more generally that

$$
\lim _{\epsilon \downarrow 0} \frac{1}{\pi} G_{X}(x, y ; E+i \epsilon)=u_{X}(x, E) u_{X}(y, E) d \rho^{X}(E) .
$$

3. $\left(2.8 / 2.8^{\prime}\right)$ are not new; they are implicit, for example, in Section II.3 of LevitanSargsjan [37].

Proof of Theorem 2.1D. (2.8) says that $|u|^{2} d \rho$ is the spectral measure for $H_{X}$ with vector $\delta_{n}$. Thus, $\int|u(n ; E)|^{2} d \rho^{X}(E)=\left(\delta_{n}, \delta_{n}\right)=1$.

Proof of Theorem 2.1C. $G_{D}(x, x ; z)$ is analytic in $\mathbb{C} \backslash[0, \infty)$ and goes to zero as $|z| \rightarrow \infty$. It follows that

$$
G_{X}(x, x ;-1)=\int \frac{|u(x, E)|^{2} d \rho^{X}(E)}{E+1} .
$$

But since $V \geq 0,\left(H_{X}+1\right)^{-1} \leq\left(H_{X}^{(0)}+1\right)^{-1}$ where $H_{X}^{(0)}$ is the operator when $V=0$. Thus,

$$
G_{X}(x, x ;-1) \leq G_{X}^{(0)}(x, x ;-1)=\frac{1}{2}\left(1 \pm e^{-2|x|}\right)
$$

by the method of images formulas for $G^{(0)}$. This proves $(2.6 \mathrm{C}(\mathrm{a}))$.

To prove $(2.6 \mathrm{C}(\mathrm{b}))$ where $x \geq 2$, pick $g$ a $C^{\infty}$ function with $0 \leq g \leq 1, g$ supported on $[-2,2]$, and $g \equiv 1$ on $[-1,1]$. Let $f(y)=g(y-x)$. Then

$$
\begin{aligned}
\int_{x-1}^{x+1}\left(u^{\prime}\right)^{2} d y & \leq \int f^{2}\left(u^{\prime}\right)^{2} d y \\
& =-\int f^{2} u^{\prime \prime} u d y-\frac{1}{2} \int\left(u^{2}\right)^{\prime}\left(f^{2}\right)^{\prime} d y \\
& =\int f(E-V) u^{2} d y+\frac{1}{2} \int\left(f^{2}\right)^{\prime \prime} u^{2} d y \\
& \leq C(1+|E|) \int_{x-2}^{x+2} u^{2} d y
\end{aligned}
$$


Thus, $(2.6(\mathrm{~b}))$ for $x \geq 2$ follows from $(2.6(\mathrm{a}))$.

A similar calculation works for $x=1$. Explicitly, pick $f$ which is supported on $[0,3)$ and $f \equiv 1$ on $[0,2]$. Because $u(0) u^{\prime}(0)=0$, the above calculations still show that

$$
\begin{aligned}
\int_{0}^{2}\left|u(y)^{\prime}\right|^{2} d y & \leq \int f(E-V) u^{2} d y+\frac{1}{2} \int\left(f^{2}\right)^{\prime \prime} u^{2} d y \\
& \leq C(1+|E|) \int_{0}^{3} u^{2} d y
\end{aligned}
$$

(2.6(b)) for $x=1$ and $x \geq 2$ imply the result for all $x$.

Remark. If $V$ is uniformly locally $L^{2}$, one can show that $(2.6 \mathrm{C}(\mathrm{b}))$ holds without the need for integrating over $y$.

\section{$\S$ 3. Criteria for A.C. Spectrum}

Our main goal in this section is to prove Theorems 1.1 and 1.2 as well as a continuum analog of Theorem 1.2. We begin with an estimate based on the Gilbert-Pearson theory and then apply the bounds of Section 2. We will then provide a new proof of the PasturIshii theorem. Finally, we present a condition for purely a.c. spectrum.

Fix $V$ and $E$. For each $\theta \in[0, \pi)$, let $\Phi_{\theta}$ be the vector formed from the solution with $(\sin \theta, \cos \theta)$ boundary conditions at 0 , that is,

$$
\Phi_{\theta}(\cdot)=T_{E}(\cdot)\left(\begin{array}{c}
\sin \theta \\
\cos \theta
\end{array}\right)
$$

and let $\Psi_{\theta}$ be $\Phi_{\pi / 2+\theta}$, that is,

$$
\Psi_{\theta}(\cdot)=T_{E}(\cdot)\left(\begin{array}{c}
\cos \theta \\
-\sin \theta
\end{array}\right)
$$

Define $u_{\theta}, v_{\theta}$ by $\Phi_{\theta}(n)=\left(\begin{array}{c}u_{\theta}(n+1) \\ u_{\theta}(n)\end{array}\right), \Psi_{\theta}(n)=\left(\begin{array}{c}v_{\theta}(n+1) \\ v_{\theta}(n)\end{array}\right)$.

The Wronskian of $u$ and $v$ is constant, that is, $\langle\Phi, J \Psi\rangle=1$ with $J=\left(\begin{array}{cc}0 & 1 \\ -1 & 0\end{array}\right)$. It follows by the Cauchy-Schwarz inequality that

$$
\left\|\Phi_{\theta}(n)\right\|\left\|\Psi_{\theta}(n)\right\| \geq 1
$$

Clearly, $\left\|\Psi_{\theta}(\cdot)\right\| \leq\left\|T_{E}(\cdot)\right\|$ by $(3.1 b)$. Let us use the symbol $\frac{1}{L} \int_{0}^{L} \cdot d x$ for the integral in the continuum case and for the sum $\frac{1}{L} \sum_{n=1}^{L}$. in the discrete case. Then

$$
\frac{1}{L} \int_{0}^{L}\left\|\Psi_{\theta}(x)\right\|^{2} d x \leq \frac{1}{L} \int_{0}^{L}\left\|T_{E}(x)\right\|^{2} d x .
$$


By (3.2),

$$
\begin{aligned}
1 & \leq\left(\frac{1}{L} \int_{0}^{L}\left\|\Phi_{\theta}(x)\right\|\left\|\Psi_{\theta}(x)\right\| d x\right)^{2} \\
& \leq\left(\frac{1}{L} \int_{0}^{L}\left\|\Phi_{\theta}(x)\right\|^{2}\right)\left(\frac{1}{L} \int_{0}^{L}\left\|\Psi_{\theta}(x)\right\|^{2} d x\right) .
\end{aligned}
$$

(3.3) and (3.4) immediately imply

\section{Lemma 3.1.}

$$
\frac{\int_{0}^{L}\left\|\Psi_{\theta}(x)\right\|^{2} d x}{\int_{0}^{L}\left\|\Phi_{\theta}(x)\right\|^{2} d x} \leq\left(\frac{1}{L} \int_{0}^{L}\left\|T_{E}(x)\right\|^{2} d x\right)^{2}
$$

Recall the definitions of Gilbert-Pearson. A solution $u_{\theta}$ is called subordinate if and only if

$$
\lim _{x \rightarrow \infty} \frac{\int_{0}^{L}\left|u_{\theta}(x)\right|^{2} d x}{\int_{0}^{L}\left|v_{\theta}(x)\right|^{2} d x}=0 .
$$

To use (3.6), we must deal with the fact that $\Phi, \Psi$ are not quite the same as $u, v$. In the discrete case, we have that

$$
\sum_{n=1}^{L+1}\left|v_{\theta}(n)\right|^{2} \leq \sum_{n=1}^{L}\left\|\Psi_{\theta}(n)\right\|^{2}
$$

while

$$
\begin{aligned}
\sum_{n=1}^{L+1}\left|u_{\theta}(n)\right|^{2} & \geq \frac{1}{2} \sum_{n=1}^{L}\left(\left|u_{\theta}(n)\right|^{2}+\left|u_{\theta}(n+1)\right|^{2}\right) \\
& \geq \frac{1}{2} \sum_{n=1}^{L}\left\|\Phi_{\theta}(n)\right\|^{2}
\end{aligned}
$$

so returning to $\int_{0}^{L} \cdot d x$ notation for the sum

$$
\frac{\int_{0}^{L+1}\left|v_{\theta}(x)\right|^{2} d x}{\int_{0}^{L+1}\left|u_{\theta}(x)\right|^{2} d x} \leq \frac{2 \int_{0}^{L}\left\|\Psi_{\theta}(x)\right\|^{2} d x}{\int_{0}^{L}\left\|\Phi_{\theta}(x)\right\|^{2} d x}
$$

so

$$
\frac{\int_{0}^{L+1}\left|v_{\theta}(x)\right|^{2} d x}{\int_{0}^{L+1}\left|u_{\theta}(x)\right|^{2} d x} \leq 2\left(\frac{1}{L} \int_{0}^{L}\left\|T_{E}(x)\right\|^{2} d x\right)^{2} .
$$

In the continuum case, one can mimic the proof of Theorem $2.1 \mathrm{C}$ to see that if $V \geq 0$, then

$$
\int_{0}^{L}\left(u_{\theta}(x)^{2}+u_{\theta}^{\prime}(x)^{2}\right) d x \leq C(1+|E|) \int_{0}^{L+1} u_{\theta}(x)^{2} d x .
$$


Thus,

$$
\begin{aligned}
\frac{\int_{0}^{L+1} v_{\theta}^{2}(x) d x}{\int_{0}^{L+1} u_{\theta}^{2}(x) d x} & \leq C(1+|E|) \frac{\int_{0}^{L+1}\left\|\Psi_{\theta}(x)\right\|^{2} d x}{\int_{0}^{L}\left\|\Phi_{\theta}(x)\right\|^{2} d x} \\
& \leq C(1+|E|)\left(\frac{1}{L} \int_{0}^{L}\left\|\Psi_{\theta}(x)\right\|^{2} d x\right)\left(\frac{1}{L} \int_{0}^{L+1}\left\|\Psi_{\theta}(x)\right\|^{2} d x\right) \\
& \leq\left(\frac{(L+1)}{L}\right)^{2} C(1+|E|)\left(\frac{1}{L+1} \int_{0}^{L+1}\left\|T_{E}(x)\right\|^{2} d x\right)^{2} .
\end{aligned}
$$

(3.6) and (3.7) imply that

Theorem 3.2. If $H$ has a subordinate solution at energy $E$, then

$$
\lim _{L \rightarrow \infty} \frac{1}{L} \int_{0}^{L}\left\|T_{E}(x)\right\|^{2} d x=\infty .
$$

Let $Q=\{E \mid H$ has a subordinate solution at energy $E\}$ and let $S_{0}=\mathbb{R} \backslash Q$. Recall $S$, the set of Theorem 1.1, is given by

$$
S=\left\{E \mid \begin{array}{l|l}
\underline{\lim } \frac{1}{L} \int_{0}^{L}\left\|T_{E}(x)\right\|^{2} d x<\infty
\end{array}\right\}
$$

Theorem 3.2 says that $Q \subset \mathbb{R} \backslash S$ so $S \subset S_{0}$. Gilbert-Pearson have shown that $S_{0}$ is the essential support of the a.c. part, $\mu_{\mathrm{ac}}$, of the spectral measure of $H_{+}$. Thus, $S \subset S_{0}$ implies that if $A \subset S$ and $|A|>0$, then $\mu_{\mathrm{ac}}(A)>0$. Theorem 1.1 thus follows from

Proposition 3.3. For a.e. E w.r.t. $\mu_{\mathrm{ac}}$, we have that $E \in S$.

Proof. In terms of the measures $d \rho^{X}$ of Section 2, let $d \mu(E)=\min \left(d \rho^{D}, d \rho^{N}\right)$ in the discrete case and $d \mu=\left(1+E^{2}\right)^{-1} \min \left(d \rho^{D}, d \rho^{N}\right)$ in the continuum case, where the min is defined viz.

$$
\min \left(\mu_{1}, \mu_{2}\right)(S)=\inf _{\substack{A, B \\ S \subset A \cup B}}\left\{\mu_{1}(A)+\mu_{2}(B)\right\}
$$

Since the singular parts of $d \rho^{D}$ and $d \rho^{N}$ are disjoint and the a.c. parts are mutually equivalent (see, e.g., [45]), $d \mu$ is equivalent to the a.c. part of the spectral measure for $H_{+}$. By (2.1) and (2.6), we have that for each $n$,

$$
\int d \mu(E)\left\|T_{E}(n)\right\|^{2} \leq 4
$$

in the discrete case and for each $x_{0} \geq 1$,

$$
\int d \mu(E) \int_{x_{0}-1}^{x_{0}+1}\left\|T_{E}(x)\right\|^{2} \leq C
$$


in the continuum case. Here $C$ is a universal constant. It follows that

$$
\int d \mu(E) G_{L}(E) \leq C
$$

where

$$
G_{L}(E)=\frac{1}{L} \sum_{n=1}^{L}\left\|T_{E}(n)\right\|^{2}
$$

in the discrete case and

$$
G_{L}(E)=\frac{1}{Q(L)} \int_{0}^{L}\left\|T_{E}(x)\right\|^{2} d x
$$

where $Q(L)$ is the smallest even integer less than $L$ (so $L / Q(L) \rightarrow 1$ as $L \rightarrow \infty$ ).

By (3.10) and Fatou's lemma, $\int d \mu(E) \underline{\lim } G_{L}(E)<\infty$, so $\underline{\lim } G_{L}(E)<\infty$ a.e. w.r.t. $d \mu$, that is, $E \in S$ for a.e. $E$ w.r.t. to $\overline{d \mu}$.

Remark. An immediate consequence of Theorem 1.1 is that if $V_{\omega}$ is an ergodic family of potentials and the Lyapunov exponent $\gamma(E)>0$ on a Borel set $T \subset \mathbb{R}$, then for a.e. $\omega, \Sigma_{\mathrm{ac}}\left(H_{\omega}\right) \cap T=\emptyset$. For by Fubini's theorem for a.e. $\omega$, for a.e. $E \in T$, we have $\lim \frac{1}{n} \ln \|T(n)\|>0$ so that a fortiori, $\lim \frac{1}{L} \sum_{n=1}^{L}\|T(n)\|^{2}=\infty$ and thus, for a.e. $\omega, S \cap T$ has zero Lebesgue measure. This result is the celebrated Ishii-Pastur theorem $[21,38,5,7]$. Note that our proof is more direct than the one that goes through the construction of exponentially decaying eigenfunctions.

To prove Theorem 1.2 (and also Theorem 1.4), we need to extend (3.9) from $T(n)$ to $T(n, m)$. As in that equation, $d \mu$ is the min of $d \rho^{N}$ and $d \rho^{D}$ which is an a.c. measure for $h_{+}$:

Theorem 3.4D. For any $n, m, \int\left\|T_{E}(n, m)\right\| d \mu(E) \leq 4$.

Proof. We have that $\|T(n, m)\| \leq\|T(n, 0)\|\|T(0, m)\|=\|T(n, 0)\|\|T(m, 0)\|$, so by the Schwarz inequality,

$$
\int\left\|T_{E}(n, m)\right\| d \mu \leq\left(\int\left\|T_{E}(n, 0)\right\|^{2} d \mu\right)^{1 / 2}\left(\int\left\|T_{E}(m, 0)\right\|^{2} d \mu\right)^{1 / 2} \leq 4
$$

by $(3.9)$.

An immediate consequence of this theorem and Fatou's lemma is

Theorem 3.5D (三 Theorem 1.2). Let $m_{j}, k_{j}$ be arbitrary sequences in $\{n \in \mathbb{Z} \mid$ $n>0\}$. Then for a.e. E in the a.c. part of the spectral measure for $h_{+}$, we have that $\underline{\lim }_{j \rightarrow \infty}\left\|T_{E}\left(m_{j}, k_{j}\right)\right\|<\infty$.

The continuum versions of these results are straightforward analogs following the above proof using (3.9C). Here $d \mu(E)=\left(1+E^{2}\right)^{-1} \min \left(d \rho^{D}(E), d \rho^{N}(E)\right)$. 
Theorem 3.4C. For each $x_{0}, y_{0}$ and a universal constant $C$,

$$
\int d \mu(E)\left[\int_{x_{0}-1}^{x_{0}+1} d x \int_{y_{0}-1}^{y_{0}+1} d y\left\|T_{E}(x, y)\right\|\right]<C .
$$

Theorem 3.5C. Let $x_{j}, y_{j}$ be arbitrary sequences in $\{x \in \mathbb{R} \mid x>0\}$. Then for a.e. $E$ in the a.c. part of the spectral measure for $H_{+}$, we have that

$$
\varliminf_{j \rightarrow \infty} \int_{x_{j}-1}^{x_{j}+1} d x \int_{y_{j}-1}^{y_{j}+1} d y\left\|T_{E}(x, y)\right\|<\infty
$$

We will need the following variant of these ideas in Section 5:

Theorem 3.6D. In the discrete case,

$$
\int d \mu(E)\left(\frac{1}{L} \sum_{m=n+1}^{n+L}\|T(m, n)\|^{2}\right)^{1 / 2} \leq 4
$$

Proof. Since $\|T(m, n)\| \leq\|T(m)\|\|T(n)\|$, we have that $\left(\frac{1}{L} \sum_{m=n+1}^{n+L}\|T(m, n)\|^{2}\right) \leq$ $\|T(n)\|\left(\frac{1}{L} \sum_{m=n+1}^{n+L}\|T(m)\|^{2}\right)^{1 / 2}$, so (3.11) follows from (3.9D) and the Schwarz inequality.

In the same way, we get

Theorem 3.6C. In the continuum case for a universal constant $C$,

$$
\int d \mu(E)\left(\int_{x_{0}-1}^{x_{0}+1} d x \frac{1}{Q(L)} \int_{x}^{x+L}\left\|T_{E}(x, y)\right\|^{2} d y\right)^{1 / 2} \leq C .
$$

Theorem 3.7 (= Theorem 1.3). Suppose that for some $x_{n} \rightarrow \infty$,

$$
\lim _{n \rightarrow \infty} \int_{a}^{b}\left\|T_{E}\left(x_{n}\right)\right\|^{p} d E<\infty
$$

for some $p>2$. Then for any boundary condition at zero, the spectral measure is purely absolutely continuous on $(a, b)$. More generally, if $W$ is an arbitrary function on $(-\infty, \infty)$ so that

(i) $W=V$ on $(0, \infty)$

(ii) $W$ is limit point at both $-\infty$ and $\infty$. 
Then $H=-\frac{d^{2}}{d x^{2}}+W$ has purely a.c. spectrum on $(a, b)$.

Proof. Fix a boundary condition $\theta$ at zero and let $u_{\theta}=(\cos (\theta), \sin (\theta))$. For any $x$, let

$$
d \mu_{x}^{\theta}(E)=\pi^{-1} d E /\left\|T_{E}(x) u_{\theta}\right\|^{2} .
$$

Then Carmona [4] proves that as $x \rightarrow \infty$,

$$
d \mu_{x}^{\theta} \rightarrow d \mu^{\theta}
$$

the spectral measure for boundary condition $\theta$ (the convergence in (3.13) is in the vague sense, i.e., it holds after smearing with continuous functions in $E$ ). Since $T$ is unimodular, $\left\|T^{-1}\right\|=\|T\|$ so $\left\|T u_{\theta}\right\| \geq\|T\|^{-1}\left\|u_{\theta}\right\|$ and thus, $d \mu_{x}^{\theta}(E)=F_{x}^{\theta}(E) d E$ with

$$
\left|F_{x}(E)\right| \leq\left\|T_{E}(x)\right\|^{2} \text {. }
$$

For the whole-line problem, Carmona proves a result similar to (3.12/3.13), but in (3.12) $\left\|T_{E}(x) u_{\theta}\right\|$ is replaced by $\left\|T_{E} u_{\theta(E)}\right\|$ with $\theta(E)$ dependent on $E$ (and $x$ ) but (3.14) still holds. The result now follows from the next lemma.

Lemma 3.8. Let $f_{n}(\lambda)$ be a sequence of functions on $(a, b) \subset \mathbb{R}$ so that for some $q>1$,

$$
\int f_{n}(\lambda)^{q} d \lambda \leq C
$$

uniformly in $n$. Suppose that $f_{n}(\lambda) d \lambda$ converge to a measure $d \mu(\lambda)$ weakly. Then $d \mu$ is purely absolutely continuous.

Proof. The ball of radius $C$ in $L^{q}$ is compact in the weak-* topology, so there exists a subsequence $f_{n(i)}$ and $f_{\infty} \in L^{p}$ so that $\int f_{n(i)}(\lambda) g(\lambda) d \lambda \rightarrow \int f_{\infty}(\lambda) g(\lambda) d \mu(\lambda)$ for all $g \in L^{q^{\prime}}$ with $q^{\prime}$ dual to $p$. Thus, $d \mu=f_{\infty} d \lambda$ is absolutely continuous.

We end this section with two remarks that shed some light on the earlier theorems in this section. The first concerns an explicit relationship between the $m$-function and the basic average $\frac{1}{L} \int_{0}^{L}\left\|T_{E}(x)\right\|^{2} d x$ which is connected with Lemma 3.1:

Proposition 3.9. We have for any $\theta$ that

$$
\operatorname{Im} m_{\theta}\left(E+i \frac{1}{L}\right) \leq(5+\sqrt{24})\left[\frac{1}{L} \sum_{n=0}^{L+1}\left\|T_{E}(n)\right\|^{2}\right]
$$

where $\left\|T_{E}(0)\right\|$ is short for 1 .

Proof. Let $u_{1}$ be the solution with $\theta$ boundary conditions normalized at $n=1$ and $u_{2}$ the solution with complementary $\left(\frac{\pi}{2}-\theta\right)$ boundary conditions. Then Jitomirskaya-Last $[23,24]$ prove that if $\|f\|_{L}=\left(\left.\sum_{n=1}^{L} f(n)\right|^{2}\right)^{1 / 2}$ and $\epsilon(L)$ is defined by

$$
\left\|u_{1}\right\|_{L}\left\|u_{2}\right\|_{L}=(2 \epsilon)^{-1}
$$


then

$$
|m(E+i \epsilon)| \leq(5+\sqrt{24}) \frac{\left\|u_{2}\right\|_{L}}{\left\|u_{1}\right\|_{L}} .
$$

If $L$ is odd, let $f=\left(u_{1}(1), u_{1}(2), \ldots, u_{1}(L-1)\right)$ and let $g=\left(u_{2}(2),-u_{2}(1), u_{2}(4),-u_{2}(3)\right.$, $\left.\ldots,-u_{2}(L-1)\right)$. Then constancy of the Wronskian implies that $\langle f, g\rangle=\frac{L}{2}$, so by the Schwarz inequality,

$$
\frac{L-1}{2} \leq\left\|u_{1}\right\|_{L}\left\|u_{2}\right\|_{L}=\frac{1}{2 \epsilon(L)} .
$$

For $L$ even, the inequality holds with $\frac{L}{2}$, so a fortiori, (3.16) holds. Thus, $\frac{1}{L} \geq \epsilon(L+1)$. Since $\operatorname{Im} m(E+i \epsilon) / \epsilon$ is monotone increasing as $\epsilon$ decreases,

$$
\begin{aligned}
\frac{\operatorname{Im} m\left(E+i L^{-1}\right)}{L^{-1}} & \leq \frac{\operatorname{Im} m(E+i \epsilon(L+1))}{\epsilon(L+1)} \\
& \leq \frac{|m(E+i \epsilon)|}{\epsilon} \leq \frac{5+\sqrt{24}}{\epsilon} \frac{\left\|u_{2}\right\|_{L+1}}{\left\|u_{1}\right\|_{L+1}} .
\end{aligned}
$$

By (3.15), $\left(\epsilon\left\|u_{1}\right\|_{L+1}\right)^{-1} \leq 2\left\|u_{2}\right\|_{L+1}$, so

$$
\frac{\operatorname{Im} m\left(E+i L^{-1}\right)}{L^{-1}} \leq 2(5+\sqrt{24})\left\|u_{2}\right\|_{L+1}^{2} .
$$

Now $\left|u_{2}(n)\right|^{2}+\left|u_{2}(n+1)\right|^{2} \leq\|T(n)\|^{2}$, so

$$
\sum_{n=0}^{L+1}\|T(n)\|^{2} \geq|u(0)|^{2}+|u(L+2)|^{2}+2\left\|u_{2}\right\|_{L+1}^{2}
$$

proving that $2\left\|u_{2}\right\|_{L+1}^{2} \leq \sum_{n=0}^{L+1}\|T(n)\|^{2}$ and the claimed inequality.

The second result concerns the fact that $\underline{\lim } \frac{1}{L} \sum_{n=1}^{L}\|T(n)\|^{2}<\infty$ says nothing about upper bounds. We claim that this sum cannot grow too fast, at least for a.e. $E$ w.r.t. $d \mu_{\text {ac }}$.

Theorem 3.10. Fix $\delta>0$. For a.e. E w.r.t. $d \mu_{\mathrm{ac}}$, we have that for any $L \geq 2$,

$$
\left(\frac{1}{L} \sum_{n=1}^{L}\left\|T_{E}(n)\right\|^{2}\right) \leq C_{E}(\log L)^{1+\delta}
$$

Remarks. 1. $(\log L)^{1+\delta}$ can be replaced by any increasing function $f(n)$ with $\sum f\left(2^{n}\right)^{-1}<$ $\infty$, for example, $(\log L)(\log (\log L))^{1+\delta}$.

2. If we replace $\left\|T_{E}(n)\right\|$ by $\|u(n ; E)\|$, this result holds for $d \mu(E)$ rather than just for $d \mu_{\mathrm{ac}}(E)$. 
Proof. Let $g_{k}(E)=2^{-k} \sum_{n=1}^{2^{k}}\left\|T_{E}(n)\right\|^{2}$. Then by $(3.9 \mathrm{D}), \int g_{k}(E) d \mu_{\mathrm{ac}}(E) \leq 4$ so $\sum_{k=1}^{\infty} k^{-1-\delta} g_{k}(E) \in L^{1}\left(d \mu_{\mathrm{ac}}\right)$, which, in particular, implies that

$$
g_{k}(E) \leq C_{E} k^{1+\delta}
$$

for a.e. $E$ w.r.t. $d \mu_{\mathrm{ac}}$.

Let $2^{k-1} \leq L \leq 2^{k}$. Then

$$
L^{-1} \sum_{n=1}^{L}\left\|T_{E}(n)\right\|^{2} \leq 2^{-k-1} \sum_{n=1}^{2^{k}}\left\|T_{E}(n)\right\|^{2} \leq 2 g_{k}(E)
$$

so (3.17) completes the proof.

\section{§. Barriers and A.C. Spectrum}

Theorem 1.2, which we proved in Section 3, is ideal for showing that barriers can prevent a.c. spectrum, an idea originally developed by Simon-Spencer [46]. In this section, we will explain how to recover their results using Theorem 1.2. Our techniques here allow one to go further since they can handle the case where $V$ goes to zero. We will illustrate this at the end of this section. A more thorough analysis of this case will be made in a forthcoming paper [36]. As the simplest example of the strategy, we recover

Theorem 4.1 ([46]). Let $h_{+}$be a Jacobi matrix on $\ell^{2}\left(\mathbb{Z}^{+}\right)$. Suppose $\overline{\lim }|V(n)|=\infty$. Then $h_{+}$has no a.c. spectrum.

Proof. Pick $n_{j}$ so $\left|V\left(n_{j}\right)\right| \rightarrow \infty$. Then

$$
T_{E}\left(n_{j}, n_{j}-1\right)=\left(\begin{array}{cc}
E-V\left(n_{j}\right) & -1 \\
1 & 0
\end{array}\right),
$$

so for all $E,\left\|T_{E}\left(n_{j}, n_{j}-1\right)\right\| \rightarrow \infty$ as $j \rightarrow \infty$. By Theorem 1.2, the a.c. spectrum must be empty.

To recover some of the other results of [46], we need bounds that show if $E$ is in the middle of a gap of size $2 \delta$, then the transfer matrix over a length $L$ has an a priori bound that grows as $L \rightarrow \infty$ in a way independent of the potential. We could obtain this using Combes-Thomas estimates with explicit constants (as in [46]) or using the periodic potential methods of $[34,35]$, but we will instead use the idea of approximate eigenfunctions. Our simple estimates can be viewed as a quantitative version of an idea of Sch'nol [42]. Basically, we will see that any solution is found to grow exponentially at a pre-assigned rate in some direction. 
Theorem 4.2. Suppose $h$ is a one-dimensional operator of the form (1.1D) on a subset $D$ of $\mathbb{Z}$ with $D_{n} \equiv\{-n,-n+1, \ldots, n-1, n\} \subset D$. Suppose that there is an operator $B$ on $\ell^{2}\left(D^{\prime}\right)$ for some $D^{\prime} \subset \mathbb{Z}$ with $D_{n} \subset D^{\prime}$ so that

(i) $\operatorname{spec}(B) \cap(E-\delta, E+\delta)=\emptyset$.

(ii) $B u=h u$ if $u$ vanishes outside $D_{n}$.

Then

(a) Any solution of $h u=E u$ obeys

$$
|u(\ell)|^{2}+\left|u(-\ell)^{2}\right| \geq \delta^{2}\left(1+\delta^{2}\right)^{\ell-1}|u(0)|^{2} \quad \text { for } \ell=1,2, \ldots, n+1
$$

and

$|u(\ell)|^{2}+|u(-\ell)|^{2} \geq \delta^{2}\left(1+\delta^{2}\right)^{\ell-2}\left(|u(0)|^{2}+|u(1)|^{2}+|u(-1)|^{2}\right) \quad$ for $\ell=2,3, \ldots, n+1$.

(b) For any vector $\varphi \in \mathbb{R}^{2}$,

$$
\|T(\ell, 0) \varphi\|^{2}+\|T(-\ell, 0) \varphi\|^{2} \geq \delta^{2}\left(1+\delta^{2}\right)^{\ell-1}\|\varphi\|^{2} \quad \text { for } \ell=1,2, \ldots, n .
$$

(c) We have that

$$
\|T(-n, n)\| \geq \frac{1}{2} \delta^{2}\left(1+\delta^{2}\right)^{n-1} .
$$

Remarks. 1. One remarkable aspect of these estimates is that they (and their multidimensional case continuum analog) are independent of $V$.

2. The point, of course, is that since $\delta>0,\left(1+\delta^{2}\right)^{\ell}$ grows to infinity as $\ell \rightarrow \infty$. We show it is exponentially fast, but that is not needed.

3. While the estimates are elegant and explicit, it is likely the exponent is not optimal. For $\delta$ small, $\left(1+\delta^{2}\right)^{n} \simeq \exp \left(n \log \left(1+\delta^{2}\right)\right) \sim \exp \left(n \delta^{2}\right)$. One would expect that $\|T(n,-n)\| \sim \exp (2 \delta n)$ for $\delta$ small (and fixed) and $n$ large.

Proof. Let $\chi_{j}$ be the characteristic function of $\{-j, \ldots, j\}$. Then

$$
\left((h-E)\left(\chi_{j} u\right)\right)(\ell)=-\delta_{j, \ell+1} u(\ell+1)-\delta_{j,-\ell-1} u(-\ell-1)
$$

so if we define

$$
a_{j} \equiv|u(j)|^{2}+|u(-j)|^{2} \quad \text { for } j=1,2, \ldots
$$

and

$$
a_{0} \equiv|u(0)|^{2}
$$

we have that

$$
\left\|(H-E)\left(\chi_{j} u\right)\right\|^{2}=a_{j+1} .
$$

Clearly,

$$
\left\|\chi_{j} u\right\|^{2}=\sum_{k=0}^{j} a_{k} .
$$


But by hypothesis (i), (ii), if $j=0,1, \ldots, n$,

$$
\left\|(H-E) \chi_{j} u\right\|^{2}=\left\|(B-E) \chi_{j} u\right\|^{2} \geq \delta^{2}\left\|\chi_{j} u\right\|^{2} .
$$

(4.5), (4.6), and (4.7) imply that

$$
\delta^{2}\left(\sum_{k=0}^{j} a_{k}\right) \leq a_{j+1}
$$

for $j=0,1,2, \ldots, n$.

It follows inductively that for $\ell=1,2, \ldots$

$$
a_{\ell} \geq \delta^{2}\left(1+\delta^{2}\right)^{\ell-1} a_{0}
$$

for (4.9) holds for $\ell=1$ (by 4.8), and if (4.9) holds for $a_{1}, \ldots, a_{j}$, then by (4.8),

$$
a_{j+1} \geq \delta^{2}\left(1+\sum_{k=1}^{j} \delta^{2}\left(1+\delta^{2}\right)^{k-1}\right) a_{0}=\delta^{2}\left(1+\delta^{2}\right)^{j} a_{0} .
$$

(4.9) is precisely (4.1).

A virtually identical inductive argument proves (4.2). (4.3) follows from (4.1) and its translate:

$$
|u(\ell+1)|^{2}+|u(-\ell+1)|^{2} \geq \delta^{2}\left(1+\delta^{2}\right)^{\ell-1}|u(1)|^{2} ; \quad \ell=1, \ldots, n .
$$

To prove (4.4), let $\alpha=\frac{1}{2} \delta^{2}\left(1+\delta^{2}\right)^{n-1}$ so that (4.3) becomes

$$
\|T(n, 0) \varphi\|^{2}+\|T(-n, 0) \varphi\|^{2} \geq 2 \alpha\|\varphi\|^{2} .
$$

If $\alpha \leq 1,(4.4)$ is trivial so suppose that $\alpha>1$. Picking any unit vector $\varphi$, we conclude that

$$
\|T(n, 0)\|^{2} \geq \alpha \quad \text { or } \quad\|T(-n, 0)\|^{2} \geq \alpha .
$$

Suppose the former. Since $T(n, 0)$ is unimodular, we can find a unit vector $\varphi_{0}$ so that $\left\|T(n, 0) \varphi_{0}\right\|=\|T(n, 0)\|^{-1}$. Thus,

$$
\left\|T(n, 0) \varphi_{0}\right\|^{2} \leq \frac{1}{\alpha}\left\|\varphi_{0}\right\|^{2} \leq \alpha\left\|\varphi_{0}\right\|^{2}
$$

because we are supposing that $\alpha>1$. Thus, by (4.10),

$$
\left\|T(-n, 0) \varphi_{0}\right\|^{2} \geq \alpha\left\|\varphi_{0}\right\|^{2} \geq \alpha^{2}\left\|T(n, 0) \varphi_{0}\right\|^{2} .
$$

It follows that

$$
\|T(n,-n)\|^{2}=\|T(-n, n)\|^{2} \geq \alpha^{2}
$$

which is (4.4).

Once we have Theorem 4.2, we immediately conclude by Theorem 1.2 that 
Theorem 4.3. Suppose that $h$ has the form (1.1D) on $\mathbb{Z}^{+}$so that there exist $x_{n} \geq n$ and $W_{n}$ on $\tilde{D}_{n} \supset\left\{x_{n}-n, \ldots, x_{n}+n\right\}$ so that

(i) $(\alpha, \beta) \cap \operatorname{spec}\left(-\frac{d^{2}}{d x^{2}}+W_{n}\right)=\emptyset$ for some boundary conditions on $\tilde{D}_{n}$.

(ii) $W_{n}(j)=V(j)$ for $j \in\left\{x_{n}-n, \ldots, x_{n}+n\right\}$.

Then $(\alpha, \beta)$ is disjoint from the a.c. spectrum of $h$.

Proof. Fix $E \in(\alpha, \beta)$. Then by Theorem 4.2,

$$
\lim _{n \rightarrow \infty}\left\|T_{E}\left(x_{n}-n, x_{n}+n\right)\right\|=\infty .
$$

It follows by Theorem 1.2 that $(\alpha, \beta)$ is disjoint from the a.c. spectrum.

With this result, one can recover the theorems in [46] that depend on gaps in the spectrum.

Before leaving the subject of Theorem 4.2, we note that (4.1) has a continuum, higherdimensional analog.

Theorem 4.4. For any $K>0$ and dimension $\nu$, there exists a universal constant $C_{\nu}(K)$ depending only on $\nu$ and $K$ so that if $V$ is in the local Kato class and there exists an operator $B$ on $L^{2}\left(\mathbb{R}^{\nu}\right)$ so that

(i) $B u=(-\Delta+V) u$, all $u \in C_{0}^{\infty}\left(D_{n}\right)$ where $D_{n}=\{x|| x \mid \leq n+1\}$

(ii) $\sigma(B) \cap(E-\delta, E+\delta)=\emptyset$

(iii) $\left\|V \chi_{\{x|| x \mid \leq n+1\}}\right\| \leq K$ where the norm is the $K_{\nu}$ Kato class norm $[7,43]$

(iv) $|E| \leq K$

then any $L_{\text {loc }}^{2}$ distributional solution of $(-\Delta+V) u=E u$ in $D_{n}$ obeys

$$
\int_{j \leq|x| \leq j+1}|u(x)|^{2} d x \geq C_{\nu}(K) \delta^{2}\left(1+C_{\nu}(K) \delta^{2}\right)^{j-2} \int_{|x| \leq 1}|u(x)|^{2} d x
$$

for $j=1,2, \ldots, n$.

Proof. Let $\chi_{j}$ be the characteristic function of $\{x|| x \mid \leq j\}$. It is fairly easy to see one can construct a sequence, $f_{j}$, of $C^{\infty}$ functions on $\mathbb{R}^{\nu}$ so that

$$
\begin{aligned}
f_{j} \chi_{j} & =\chi_{j} \\
f_{j} \chi_{j+1} & =f_{j}
\end{aligned}
$$

and

$$
\sup _{j}\left\|D^{\alpha} f_{j}\right\| \equiv d_{\alpha}<\infty
$$

for each multi-index $\alpha$.

We claim that with $H=-\Delta+V$,

$$
\left\|(H-E) f_{j} u\right\|^{2} \leq C_{\nu}(K)^{-1}\left\|\left(\chi_{j+1}-\chi_{j}\right) u\right\|^{2} .
$$


Accepting this for a moment, we will prove (4.8). We have for $j \leq n-1$,

$$
\left\|(H-E) f_{j} u\right\|^{2}=\left\|(B-E) f_{j} u\right\|^{2} \geq \delta^{2}\left\|f_{j} u\right\|^{2} \geq \delta^{2}\left\|\chi_{j} u\right\|^{2} .
$$

Thus with $a_{j}=\left\|\left(\chi_{j+1}-\chi_{j}\right) u\right\|^{2}$, we see that

$$
C_{\nu} \delta^{2}\left(\sum_{1}^{j} a_{\ell}\right) \leq a_{j+1}
$$

so that as in the proof of Theorem 4.2,

$$
a_{j} \geq C_{\nu} \delta^{2}\left(1+C_{\nu} \delta^{2}\right)^{j-2} a_{1}
$$

which is (4.11).

To prove (4.13), notice that

$$
(H-E) f_{j} u=\left(-\Delta f_{j}\right) u+2\left(\nabla f_{j}\right) \cdot \nabla u
$$

SO

$$
\left\|(H-E) f_{j} u\right\|^{2} \leq 2\left\|\left(-\Delta f_{j}\right) u\right\|^{2}+8\left\|\left(\nabla f_{j}\right) \cdot \nabla u\right\|^{2} .
$$

By Theorem C.2.2 of [43], we can bound $\|\nabla f \cdot \nabla u\|^{2}$ by a constant $C_{1}$ (depending on $K)$ times $\left\|\left(\chi_{j-1}-\chi_{j}\right) u\right\|^{2}$ so by (4.14), we have the estimate (4.13).

Theorem 4.5. Fix $\alpha<\frac{1}{2}$ and let $\left\{a_{n}\right\}_{n=1}^{\infty}$ be identically independently distributed random variables with distribution $\frac{1}{2} \chi_{[-1,1]}(x) d x$. Then there exists $N_{1}<N_{2}<\cdots$ so that for any $m_{1}, \ldots, m_{n}, \cdots \geq 0$ and a.e. $\left\{a_{n}\right\}$ the potential on $\mathbb{Z}^{+}$:

$$
V(n)= \begin{cases}0 & n \leq m_{1} \\ \left(n-m_{1}\right)^{-\alpha} a_{n} & m_{1}<n \leq m_{1}+N_{1} \\ 0 & m_{1}+N_{1}<n \leq m_{1}+N_{1}+m_{2} \\ \vdots & \\ \left(n-m_{1}-N_{1}-\cdots-m_{j}\right)^{-\alpha} a_{n} & m_{1}+\cdots+N_{j-1}+m_{j}<n \leq m_{1}+\cdots+N_{j} \\ 0 & m_{1}+\cdots+N_{j}<n \leq m_{1}+\cdots+N_{j}+m_{j}\end{cases}
$$

has no a.c. spectrum.

Remark. The choice can be made so that by Theorem 1.6, there is no point spectrum, that is, so the spectrum is purely singular continuous.

Proof. Let $\tilde{T}_{E}(0, n)$ be the transfer matrix for the power-decaying potential $n^{-\alpha} a_{n}$. By [44], for a.e. $\left\{a_{n}\right\}$ and a.e. $E \in[-2,2]$,

$$
\lim _{n \rightarrow \infty}\left\|\tilde{T}_{E}(0, n)\right\|=\infty
$$


Let $A_{\ell}^{(1)}=\left\{E \mid \inf _{n \geq \ell}\left\|\tilde{T}_{E}(0, n)\right\| \geq 1\right\}$. By $(4.15)\left|[-2,2] \backslash A_{\ell}\right| \downarrow 0$ as $\ell \rightarrow \infty$ so we can pick $N_{1}$ so that $[-2,2] \backslash\left|A_{N_{1}}^{(1)}\right| \leq 2^{-1}$. Now inductively pick $N_{j}$ given $N_{1}, \ldots, N_{j-1}$ so if

$$
A_{\ell}^{(j)}=\left\{E \mid \inf _{n \geq \ell}\left\|\tilde{T}_{E}\left(N_{1}+\cdots+N_{j-1}, N_{1}+\cdots+N_{j-1}+n\right)\right\| \geq n\right\}
$$

then $[-2,2] \backslash\left|A_{N_{j}}^{(j)}\right| \leq 2^{-j}$.

For this choice of $N_{j}$ 's, the theorem holds since for a.e. $E, E \in A_{N_{j}}^{(j)}$ for all large $j$ and thus for such $E,\left\|T_{E}\left(m_{1}+\cdots+N_{j-1}+m_{j}, m_{1}+\cdots+m_{j}+N_{j}\right)\right\| \geq j$. Theorem 3.5 implies $\sigma_{\mathrm{ac}}=\emptyset$.

[29] will have a much more effective analysis of this type of example.

\section{$\S 5$. Semicontinuity of the A.C. Spectrum}

In this section, we will prove Theorem 1.4. Consider first the discrete case. Pick $n_{j}$ so $V\left(n+n_{j}\right) \rightarrow W(n)$ as $j \rightarrow \infty$ for each $n$. Let $T_{V}$ (resp. $T_{W}$ ) denote the transfer matrix for the Jacobi matrix with $V$ (resp. $W$ ) along the diagonal. By Theorem 3.6D,

$$
\int d \mu_{V}(E)\left(\frac{1}{L} \sum_{m=n_{j}+1}^{n_{j}+L}\left\|T_{V}\left(m, n_{j}\right)\right\|^{2}\right)^{1 / 2} \leq 4
$$

where $d \mu_{V}(E)$ is a measure equivalent to the a.c. part of the spectral measure for $V$. Since $V\left(n+n_{j}\right) \rightarrow W(n)$ as $j \rightarrow \infty$, we have that

$$
T_{V}\left(n_{j}+m, n_{j}\right) \rightarrow T_{W}(m, 0)
$$

so (5.1) implies that

$$
\int d \mu_{V}(E)\left(\frac{1}{L} \sum_{m=1}^{L}\left\|T_{W}(m, 0)\right\|^{2}\right)^{1 / 2} \leq 4
$$

It follows by Fatou's lemma that for a.e. $E$ with respect to $d \mu_{V}(E)$, we have

$$
\underline{\lim } \frac{1}{L} \sum_{m=1}^{L}\left\|T_{W}(m, 0)\right\|^{2}<\infty
$$

Such $E$ are thus a.e. in $\Sigma_{\text {ac }}\left(h_{0}+W\right)$, that is, $\Sigma_{\text {ac }}\left(h_{0}+V\right) \subset \Sigma_{\text {ac }}\left(h_{0}+W\right)$ as claimed.

The proof in the continuum case is similar, except that we use Theorem $3.6 \mathrm{C}$ in place of Theorem 3.6D. 
We note that the notion of right/left limits, which enters in Theorem 1.4, is in the spirit of the notion of limit class introduced by Davies-Simon [9].

\section{$\S 6$. Consequences of Semicontinuity of the A.C. Spectrum}

Let $(\Omega, T, \mu)$ be a metric ergodic process, that is, $T$ is a continuous invertible bijection from $\Omega \rightarrow \Omega$ with $\Omega$ a compact metric space (recall that any separable compact space is metrizable) and $\mu$ a probability measure with support $\mu=\Omega$.

Definition. A point $\omega_{0} \in \Omega$ is called right prototypical if and only if $\left\{T^{n} \omega_{0} \mid n \geq 0\right\}$ is dense in $\Omega$, and left prototypical if and only if $\left\{T^{n} \omega_{0} \mid n \leq 0\right\}$ is dense in $\Omega$. If $\omega_{0}$ is both left and right prototypical, we say it is prototypical.

The ergodic theorem implies that a.e. $\omega_{0} \in \Omega$ is prototypical. Fix a continuous function $f: \Omega \rightarrow \mathbb{R}$ and let $h_{\omega}$ on $\ell^{2}(\mathbb{Z})$ be defined by $\left(h_{\omega} u\right)(n)=u(n+1)+u(n-1)+f\left(T^{n} \omega\right) u(n)$.

Theorem 6.1. The essential support of the a.c. spectrum of $h_{\omega}$ is the same for all prototypical points and is of multiplicity 2. Moreover, for any prototypical $\omega_{0}$ and any $\omega \in \Omega$, we have $\Sigma_{\mathrm{ac}}\left(h_{\omega_{0}}\right) \subset \Sigma_{\mathrm{ac}}\left(h_{\omega}\right)$.

Proof. Let $h_{\omega}^{ \pm}$be the operators on $\ell^{2}( \pm n \geq 1)$ with $u(0)=0$ boundary conditions. By general principles (see, e.g., Davies-Simon [8]), the restriction of $h_{\omega}$ to its a.c. subspace is unitarily equivalent to the restriction of $h_{\omega}^{+} \oplus h_{\omega}^{-}$to its a.c. subspace. Thus, the theorem follows from

(i) if $\omega_{0}$ is prototypical and is $\omega$ arbitrary, then $\Sigma_{\mathrm{ac}}\left(h_{\omega_{0}}^{ \pm}\right) \subset \Sigma_{\mathrm{ac}}\left(h_{\omega}^{ \pm}\right)$

(ii) for prototypical $\omega_{0}, \Sigma_{\mathrm{ac}}\left(h_{\omega_{0}}^{+}\right)=\Sigma_{\mathrm{ac}}\left(h_{\omega_{0}}^{-}\right)$

for (i) implies equality if both $\omega_{0}$ and $\omega$ are prototypical and (ii) implies multiplicity 2 .

To prove (i), pick $n_{j} \rightarrow \infty$ so $T^{+n_{j}} \omega_{0} \rightarrow \omega$. Then, since $V_{T^{+m} \omega_{0}}(n)=f\left(T^{n+m} \omega_{0}\right)=$ $V_{\omega_{0}}(n+m)$, we have that $V_{\omega_{0}}\left(\cdot+n_{j}\right) \rightarrow V_{\omega}(\cdot)$ so (i) follows from Theorem 1.4.

To prove (ii), let $\omega_{0}$ be prototypical and $\omega \neq \omega_{0}$, also prototypical. Pick $n_{j} \rightarrow \infty$ so $T^{+n_{j}} \omega_{0} \rightarrow \omega$. Fix $L$ and use the fact that $\|T(n, m)\|=\|T(m, n)\|$ (since $T$ is unimodular) to note that

$$
\begin{aligned}
\frac{1}{L} \sum_{m=1}^{L}\left\|T_{\omega}(-1,-m)\right\|^{2} & =\frac{1}{L} \sum_{m=1}^{L}\left\|T_{\omega}(-m,-1)\right\|^{2} \\
& =\lim \frac{1}{L} \sum_{m=1}^{L}\left\|T_{\omega_{0}}\left(n_{j}-m, n_{j}-1\right)\right\|^{2}
\end{aligned}
$$

so by Theorem 3.6D,

$$
\int d \mu_{\omega_{0}}(E)\left(\frac{1}{L} \sum_{m=1}^{L}\left\|T_{\omega}(-1,-m)\right\|^{2}\right)<4
$$


where $d \mu_{\omega_{0}}(E)$ is an a.c. measure for $h_{\omega_{0}}^{+}$. Thus, as in the last section, $\Sigma_{\text {ac }}\left(h_{\omega}^{-}\right) \supset$ $\Sigma_{\mathrm{ac}}\left(h_{\omega_{0}}^{+}\right)$. By symmetry, (ii) holds.

Remark. That the typical a.c. spectrum is of multiplicity 2 is a result of Deift-Simon [10] proven using Kotani theory. Our proof is different.

If $V$ is almost periodic, then every $\omega \in \Omega$ is prototypical. Thus, Theorem 6.1 implies Theorem 1.5. More generally, if $(T, \Omega, \mu)$ is minimal (or if it is strictly ergodic which implies minimal), then every $\omega \in \Omega$ is prototypical, and we see that the a.c. spectrum is constant (rather than just a.e. constant) on $\Omega$.

Example. Consider the sequence $V_{1}, V_{2}, V_{3}, \ldots$ given by

$$
0,1,0,0,0,1,1,0,1,1,0,0,0,0,0,1,0,1,0, \ldots
$$

defined as follows. For two finite sequences of 0 's and 1's of length $n$, say $w_{1}, \ldots, w_{n}$, and $\tilde{w}_{1}, \ldots, \tilde{w}_{n}$, say $w<\tilde{w}$, if and only if

$$
w_{1}=\tilde{w}_{1}, \ldots, w_{j}=\tilde{w}_{j}, \quad w_{j+1}<\tilde{w}_{j+1}
$$

With this order, the sequences of length $n$ are well-ordered, for example,

$$
(0)<(1), \quad(0,0)<(0,1)<(1,0)<(1,1), \quad(0,0,0)<(0,0,1)<(0,1,0)<\cdots .
$$

$V$ is obtained by placing the two sequences of length 1 in order, then the four sequences of length 2, etc. Clearly, $V$ is prototypical for a Bernoulli model. By Furstenberg's theorem, that model has no a.c. spectrum, so $V$ is an explicit sequence for which we know that $\sigma_{\mathrm{ac}}\left(h_{0}+V\right)=\emptyset$.

Another consequence of Theorem 6.1 is a new proof of the Kotani support theorem:

Theorem 6.2. Let $\Omega$ be the compact metric space of sequences $V_{n}$ with $\left|V_{n}\right| \leq a$ with the product topology. Let $f: \Omega \rightarrow \mathbb{R}$ by $f(V)=V_{0}$ and $T: \Omega \rightarrow \mathbb{R}$ by $(T V)_{n}=V_{n+1}$. Let $\mu_{1}, \mu_{2}$ be two measures on $\Omega$ under which $T$ is ergodic. Let $\Sigma_{i}$ be the essential support of the a.c. spectrum of the prototypical $h_{\omega}$ for the process $\left(\operatorname{supp}\left(\mu_{i}\right), T, \mu_{i}\right)$. If $\operatorname{supp}\left(\mu_{1}\right) \subset \operatorname{supp}\left(\mu_{2}\right)$, then $\Sigma_{1} \supset \Sigma_{2}$.

Proof. Let $\omega_{i} \in \operatorname{supp}\left(\mu_{i}\right)$ be $\mu_{i}$-prototypical. Since $\omega_{1} \in \operatorname{supp}\left(\mu_{2}\right)$, Theorem 6.1 implies $\Sigma\left(h_{\omega_{2}}\right) \subset \Sigma\left(h_{\omega_{1}}\right)$.

Remark. In a sense, Theorem 1.4 is a deterministic version of the Kotani support theorem, so it is not surprising that it implies the Kotani theorem.

While we have stated these theorems in this section for the discrete case, they all extend easily to the continuum case. 


\section{$\S$ 7. The Potential $\lambda \cos \left(n^{\beta}\right), \beta>1$}

Jacobi matrices with potentials of the form $V(n) \equiv \lambda \cos \left(n^{\beta}\right)$, where $\lambda, \beta$ are real parameters with $\beta>1$, had been studied numerically and heuristically by GriniastyFishman [18] and Brenner-Fishman [3]. The particular case $1<\beta<2$ had been studied in more detail by Thouless [48]. The numerical evidence indicates that for $\beta \geq 2$, such potentials exhibit "localization" with the same Lyapunov exponents as those of random potentials (Anderson model) with the same coupling. The case $1<\beta<2$ is different, and far less conclusive. One still expects "localization" away from $E=0$ (the center of the spectrum) and for large $\lambda$, but the Lyapunov exponents are smaller and seem to vanish for $E=0$ and small $\lambda$. Mathematical results exist for the case where $\beta$ is an integer and $\lambda$ is large (larger than 2 , to be precise), in which case it is known [16,22] that there is no absolutely continuous spectrum. More precisely, for every polynomial $p(n)$ with a leading coefficient that is an irrational multiple of $\pi$, it is known that $\lambda \cos (p(n))$ can be obtained as a realization (an element) of an ergodic family of potentials coming from a suitable ergodic transformation on the $d$-dimensional torus [6] (with $d$ the degree of $p(n)$ ); and that the corresponding ergodic families have only positive Lyapunov exponents as long as $\lambda>2[16,22]$. Further, the corresponding ergodic families are minimal [14], and so it follows from our Theorem 6.1 that every realization of such a family has no absolutely continuous spectrum. We note that this is also true for the case $\beta=1$, where the absence of a.c. spectrum follows from earlier results [2]. Our purpose in this section is to extend these results to cases where $\beta$ is not an integer. We discuss half-line problems here, and denote by $h_{0}^{+}$the free Laplacian on $\ell^{2}\left(\mathbb{Z}^{+}\right)$. The results are also valid for full-line problems if we replace $n$ by $|n|$. We shall prove the following:

Theorem 7.1. For any $\lambda>2$ and $\beta>1, \Sigma_{\mathrm{ac}}\left(h_{0}^{+}+\lambda \cos \left(n^{\beta}\right)\right)=\emptyset$.

Remarks. 1. $\cos (\cdot)$ in the above theorem can be replaced by any real analytic function $f(\cdot)$ of period $2 \pi$, in which case the theorem would hold for $\lambda$ "large enough." This follows from the argument below combined with the results of Goldsheid-Sorets [16]. The explicit $\lambda>2$ for the $\cos (\cdot)$ case is due to Jitomirskaya [22].

2. The result is actually also more general in the sense that one can replace $n^{\beta}$ by, for example, $\sum_{j=1}^{k} a_{j} n^{\beta_{j}}$, where $k$ is any positive integer, $\beta_{j}>0$ for each $j$, and the $a_{j}$ 's are some real numbers (except if the largest $\beta_{j}$ is an integer, in which case we would need some further condition, such as that the corresponding $a_{j}$ would be an irrational multiple of $\pi$ ).

3. For $1<\beta<2$, the result also follows for $\lambda=2$, by results of Helffer-Sjöstrand [20] and Last [33].

Proof. Let $\lambda>2$. We only need to consider the case where $\beta$ is not an integer. Fix $k<\beta<k+1$, where $k$ is an integer, and consider $(n+m)^{\beta}$ where $n$ is large and $m \ll n$. By writing $(n+m)^{\beta}=n^{\beta}(1+n / m)^{\beta}$ and expanding $(1+n / m)^{\beta}$ as a Taylor series, we obtain:

$$
(n+m)^{\beta}=\sum_{\ell=0}^{\infty} a_{\ell} n^{\beta-\ell} m^{\ell}=\sum_{\ell=0}^{k} a_{\ell} n^{\beta-\ell} m^{\ell}+O\left(n^{\beta-k-1} m^{k+1}\right),
$$


where $a_{0}=1, a_{\ell}=(1 / \ell !) \prod_{j=0}^{\ell-1}(\beta-j)$ for $\ell \geq 1$. Let $b_{\ell, n}=\left\langle a_{\ell} n^{\beta-\ell} / 2 \pi\right\rangle$ where $\langle\cdot\rangle$ denotes fractional part (i.e., $\langle x\rangle=x-[x])$. Since $(n+1)^{\beta-k}-n^{\beta-k} \rightarrow 0,\left\{b_{k, n}\right\}_{n=1}^{\infty}$ is clearly dense in $[0,1]$ and we can pick a convergent subsequence $\left\{n_{j}\right\}$ so that $b_{k, n_{j}} \rightarrow b_{k, \infty} \equiv b_{k}$, where $b_{k}$ is an irrational. Moreover, by compactness, we can find a subsequence of that for which $b_{\ell, n_{j}} \rightarrow b_{\ell}$ for all $\ell \leq k$, where the $b_{\ell}$ 's (for $\ell<k$ ) are some numbers in [0,1]. For the resulting polynomial $p(n) \equiv \sum_{\ell=0}^{k} b_{\ell} n^{\ell}$ we see from $(7.1)$ that $\langle p(n) / 2 \pi\rangle$ is a pointwise limit of translations of $\left\langle n^{\beta} / 2 \pi\right\rangle$. Thus, the potential $\lambda \cos (p(n))$ is a right limit of $\lambda \cos \left(n^{\beta}\right)$, and by Theorem 1.4, we have $\Sigma_{\mathrm{ac}}\left(h_{0}^{+}+\lambda \cos \left(n^{\beta}\right)\right) \subset \Sigma_{\mathrm{ac}}\left(h_{0}^{+}+\lambda \cos (p(n))\right)=\emptyset$ (where the last equality follows from the discussion above).

In the above proof we only needed to show that some fixed realization of a suitable ergodic process is obtained as a limit of translations of $\left\langle n^{\beta} / 2 \pi\right\rangle$. However, since the underlying ergodic systems are minimal, it follows that translations of that realization are themselves dense in the ergodic family. Thus, one sees that we can actually obtain every realization as such a limit. We can combine this with Kotani's result [32] — that ergodic potentials taking finitely many values have no absolutely continuous spectrum (unless they are periodic) — to show that if $f(\cdot)$ is any real periodic piecewise constant function on the line (with only finitely many discontinuities per period), then for any $\beta>1$ that is not an integer, $\Sigma_{\mathrm{ac}}\left(h_{0}^{+}+f\left(n^{\beta}\right)\right)=\emptyset$. The proof here is very similar to that of Theorem 7.1, except that we need to choose a realization that does not take values in any of the points where $f$ is discontinuous.

Finally, we would like to discuss the special case $1<\beta<2$ and to explain how one could prove $\Sigma_{\mathrm{ac}}\left(h_{0}^{+}+\lambda \cos \left(n^{\beta}\right)\right)=\emptyset$ also for $\lambda<2$, if one could prove that "Hofstadter's butterfly has wings." Noting that we could choose the largest order coefficient $b_{k}$ in the proof of Theorem 7.1 at will, we obtain for $1<\beta<2$ :

Proposition 7.2. $\Sigma_{\mathrm{ac}}\left(h_{0}^{+}+\lambda \cos \left(n^{\beta}\right)\right) \subset \cap_{\alpha \in F} \Sigma_{\mathrm{ac}}\left(h_{0}+\lambda \cos (\pi \alpha n)\right)$ for any countable set $F$ of irrational $\alpha$ 's.

$\Sigma_{\mathrm{ac}}\left(h_{0}^{+}+\lambda \cos \left(n^{\beta}\right)\right)=\emptyset$ would thus follow, if we could prove the following:

Conjecture. Fix $\lambda \neq 0$ and $E_{0}$ real. Then there exists $\delta>0$ and irrational $\alpha$ with $\sigma\left(h_{0}+\lambda \cos (\pi \alpha n)\right) \cap\left(E_{0}-\delta, E_{0}+\delta\right)=\emptyset$.

Intuitively, this conjecture comes from the fact that for $\lambda$ small, $h_{0}+\lambda \cos (\pi \alpha n)$ should have a gap about the energy $E=2 \cos (\pi \alpha)$. In numerical drawings of Hofstadter-like butterflies for various values of $\lambda[19]$, one indeed sees those "stripes" appear for small $\lambda$, and then broaden and get more structure as $\lambda$ increases, up to the critical point $\lambda=2$ where they form the wings of the famous Hofstadter butterfly. Unfortunately, we do not know how to prove that these wings exist.

\section{$\S 8$. Transfer Matrices and Bound States}

In this section, we will prove Theorem 1.7. As noted already, Theorem 1.6 is motivation for considering $\sum_{n=1}^{\infty}\left\|T_{E}(n)\right\|^{-2}$ as an indicator of bound states. If it is infinite, $h u=E u$ has no solution $L^{2}$ at infinity. 
Example 1. Take $V=0$ and $E=2$. Then $h u=E u$ has the solutions

$$
u(n)=c_{1}+c_{2} n,
$$

none of which are $\ell^{2}$. But

$$
T_{E}(n)=\left(\begin{array}{cc}
n+1 & -n \\
n & 1-n
\end{array}\right)
$$

has $\left\|T_{E}(n)\right\|=\sqrt{2} n+0(1)$ and thus $\sum_{n+1}^{\infty}\left\|T_{E}(n)\right\|^{-2}<\infty$. We see that $\sum_{n=1}^{\infty}\left\|T_{E}(n)\right\|^{-2}$ $<\infty$ does not imply that there is an $\ell^{2}$ solution.

Example 2. Let $V(n)=c_{0} n^{-2}, n=1,2, \ldots$ with $c_{0}<\frac{1}{4}$ and $E=2$. Then standard arguments (variation of parameters) show that there are two solutions $u_{ \pm}(n)$ with

$$
u_{ \pm}(n) \sim n^{\alpha_{ \pm}}
$$

with $\alpha_{ \pm}$the roots of $\alpha(\alpha-1)+c_{0}=0$, that is,

$$
\alpha_{ \pm}=\frac{1}{2} \pm \sqrt{\frac{1}{4}-c_{0}} .
$$

We see that $\|T(n)\| \sim C n^{\alpha_{+}}$, so since $\alpha_{+}>\frac{1}{2}, \sum_{n}\|T(n)\|^{-2}<\infty$. But if $c_{0}>0$, there is no bounded solution. Thus, $\sum_{n}\|T(n)\|^{-2}<\infty$ need not even imply that there is a bounded solution!

The following (note (8.5) and (8.7)) includes the first part of Theorem 1.7 as a special case. Its proof just abstracts Ruelle [41]:

Theorem 8.1. Let $A_{1}, A_{2}, \ldots$ be unimodular $2 \times 2$ real matrices and let $T(n)=A_{n} A_{n-1}$ $\ldots A_{1}$. Suppose that

$$
\sum_{n=1}^{\infty} \frac{\left\|A_{n+1}\right\|^{2}}{\|T(n)\|^{2}}<\infty .
$$

Then there is a unit vector $u \in \mathbb{R}^{2}$ so that for any other unit vector $v \in \mathbb{R}^{2}$, we have

$$
\frac{\|T(n) u\|}{\|T(n) v\|} \rightarrow 0
$$

Proof. Let $t(n)=\|T(n)\|$ and $a(n)=\left\|A_{n}\right\|$. Since $|T(n)|$ is self-adjoint and unimodular, it has eigenvalues $t(n)$ and $t(n)^{-1}$. Thus, taking $u_{\theta}=\left(\begin{array}{c}\cos (\theta) \\ \sin (\theta)\end{array}\right)$ we see there exists $\theta_{n}$ so that

$$
\left\|T(n) u_{\theta}\right\|^{2}=t(n)^{2} \sin ^{2}\left(\theta-\theta_{n}\right)+t(n)^{-2} \cos ^{2}\left(\theta-\theta_{n}\right)
$$

for pick $\theta_{n}$ so that $|T(n)| u_{\theta_{n}}=t(n)^{-1} u_{\theta_{n}}$. Now by (8.3) for $n+1$,

$$
\begin{aligned}
t(n+1)^{2} \sin ^{2}\left(\theta_{n}-\theta_{n+1}\right) & \leq\left\|T(n+1) u_{\theta_{n}}\right\|^{2} \\
& \leq a(n+1)^{2}\left\|T(n) u_{\theta_{n}}\right\|^{2} \\
& =a(n+1)^{2} t(n)^{-2} .
\end{aligned}
$$


Since $A_{n+1}$ is unimodular, $t(n)=\|T(n)\| \leq\|T(n+1)\|\left\|A_{n+1}^{-1}\right\|=t(n+1) a(n+1)$ so

$$
t(n)^{2} \sin ^{2}\left(\theta_{n}-\theta_{n+1}\right) \leq a(n+1)^{4} t(n)^{-2} .
$$

Since $\sin ^{2}(x) \geq\left(\frac{2 x}{\pi}\right)^{2}$, we see that

$$
\left|\theta_{n}-\theta_{n+1}\right| \leq \frac{\pi}{2} \frac{a(n+1)^{2}}{t(n)^{2}}
$$

Thus, (8.1) implies

$$
\sum_{n}\left|\theta_{n}-\theta_{n+1}\right|<\infty
$$

So if (8.1) holds, $\theta_{n}$ has a limit $\theta_{\infty}$ and

$$
\left|\theta_{n}-\theta_{\infty}\right| \leq \frac{\pi}{2} \sum_{m=n}^{\infty}\left[\frac{a(m+1)^{2}}{t(m)^{2}}\right] .
$$

Let $u_{\infty}=u_{\theta_{\infty}}$ and $v_{\infty}=u_{\pi / 2+\theta_{\infty}}$. Since $\theta_{n}-\theta_{\infty} \rightarrow 0$, for $n$ large enough, we have by (8.3) that

$$
\left\|T(n) v_{\infty}\right\|^{2} \geq \frac{1}{2} t(n)^{2}
$$

On the other hand, by (8.3) again,

$$
\left\|T(n) u_{\infty}\right\|^{2} \leq t(n)^{2}\left(\theta_{n}-\theta_{\infty}\right)^{2}+t(n)^{-2} .
$$

(8.6) and (8.7) imply that

$$
\frac{\left\|T(n) u_{\infty}\right\|^{2}}{\left\|T(n) v_{\infty}\right\|^{2}} \leq 2\left(\theta_{n}-\theta_{\infty}\right)^{2}+2 t(n)^{-4} \rightarrow 0
$$

since $a(n+1) \geq 1$ and (8.1) imply that $t(n) \rightarrow \infty$. From this, (8.2) follows.

The following includes the second part of Theorem 1.7 as a special case (where $a(n)$ is bounded):

Theorem 8.2. Under the hypothesis of Theorem 8.1, suppose that we also have that

$$
\sum_{m=1}^{\infty}\|T(m)\|^{2}\left(\sum_{n=m}^{\infty} \frac{\|A(n+1)\|^{2}}{\|T(n)\|^{2}}\right)^{2}<\infty .
$$

Then there is a unit vector $u_{\infty}$ with

$$
\sum_{n=1}^{\infty}\left\|T(n) u_{\infty}\right\|^{2}<\infty
$$

Proof. Using (8.7) and (8.5), we see that $\sum_{n=1}^{\infty}\left\|T(n) u_{\infty}\right\|^{2}<\infty$ if (8.8) holds and if $\sum_{n} t(n)^{-2}<\infty$. But since $a(n) \geq 1,(8.1)$ implies that $\sum_{n} t(n)^{-2}<\infty$. 
Example 3. Suppose that $\|A(n)\|$ is bounded and $t(n) \sim n^{\gamma}$ in the sense that $C_{-} n^{\gamma} \leq$ $t(n) \leq C_{+} n^{\gamma}$. Then (8.1) requires $\gamma>\frac{1}{2}$ while (8.4) requires $\gamma>\frac{3}{2}$. Notice in Example 2, $\gamma=\alpha_{+}$while an $\ell^{2}$ solution requires $\alpha_{-}<-\frac{1}{2}$. Since $\alpha_{+}=1-\alpha_{-}$, Example 2 provides an example with $\gamma=\frac{3}{2}$ where there is no $\ell^{2}$ solution (namely, take $c_{0}=-\frac{3}{4}$ ). Thus, $\gamma>\frac{3}{2}$ is best possible!

If one has control over the limit of $\ln \|T(n)\|$, one can say more:

Theorem 8.3. Suppose that the hypotheses of Theorem 8.1 hold and that

$$
\lim _{n \rightarrow \infty} \frac{\ln \|T(n)\|}{f(n)}=1
$$

and

$$
\lim _{n \rightarrow \infty} \frac{\ln \|A(n)\|}{f(n)}=0,
$$

where $f(n)$, a monotone increasing function, is such that

$$
\sum e^{-\epsilon f(n)}<\infty
$$

for any $\epsilon>0$. Then the $u_{\infty}$ of Theorem 8.2 obeys

$$
\lim _{n \rightarrow \infty} \frac{\ln \left\|T(n) u_{\infty}\right\|}{f(n)}=-1 .
$$

Proof. By (8.5) and (8.7), for any $\epsilon>0$, for $n$ large

$$
\begin{aligned}
\left\|T(n) u_{\infty}\right\|^{2} & \leq e^{-2(1-\epsilon) f(n)}+\left(\frac{\pi}{2}\right)^{2}\left(\sum_{m=n}^{\infty} \frac{e^{2 \epsilon f(m)}}{e^{2(1-\epsilon) f(m)}}\right)^{2} e^{2(1+\epsilon) f(n)} \\
& \leq e^{-2(1-\epsilon) f(n)}+\left(\frac{\pi}{2}\right)^{2} e^{-(2-11 \epsilon) f(n)}\left[\sum_{m=n}^{\infty} e^{-\epsilon f(m)}\right]
\end{aligned}
$$

so

$$
\varlimsup \frac{\left\|T(n) u_{\infty}\right\|}{f(n)} \leq-1 .
$$

On the other hand, $\|T(n) u\|_{\infty} \geq\|T(n)\|^{-1}$ implies

$$
\underline{\lim } \frac{\left\|T(n) u_{\infty}\right\|}{f(n)} \geq-1 .
$$

Typical cases of this theorem are $f(n)=n^{\alpha} ; f(n)=n$ is Ruelle's theorem. For the case $f(n)=\ln (n)$ where (8.9) fails, we have 
Theorem 8.4. Suppose the hypotheses of Theorem 8.1 hold and that

$$
\begin{aligned}
& \lim _{n \rightarrow \infty} \frac{\ln \|T(n)\|}{\ln n}=\gamma \\
& \lim _{n \rightarrow \infty} \frac{\ln \|A(n)\|}{\ln n}=0
\end{aligned}
$$

where $\gamma>\frac{1}{2}$. Then

$$
\varlimsup_{n \rightarrow \infty} \frac{\ln \left\|T(n) u_{\infty}\right\|}{\ln n} \leq 1-\gamma
$$

while

$$
\varliminf_{n \rightarrow \infty} \frac{\ln \left\|T(n) u_{\infty}\right\|}{\ln n} \geq-\gamma .
$$

Proof. As in the last theorem, (8.11) is a consequence of $\left\|T(n) u_{\infty}\right\| \geq\|T(n)\|^{-1}$. To get (8.10), we use (8.5), (8.7) to see that for any $\epsilon>0$,

$$
\begin{aligned}
\left\|T(n) u_{\infty}\right\|^{2} & \leq n^{-2 \gamma+\epsilon}+\left(\sum_{m=n}^{\infty} m^{-2 \gamma+\epsilon}\right)^{2} n^{2 \gamma+\epsilon} \\
& \leq n^{-2 \gamma+\epsilon}+C n^{2-2 \gamma+2 \epsilon} .
\end{aligned}
$$

Example 2 shows there are cases where the limit is $1-\gamma$. [29] has examples where the limit is $-\gamma$.

The ideas of this section can be applied to certain continuum problems by sampling the wave function at a discrete set of points.

\section{Appendix: BGK Eigenfunction Expansions}

The proofs of the estimates in Section 2 are one-dimensional, relying on the relation between Green's functions and $m$-functions. Our goal in this appendix is to discuss an alternate proof which extends to higher dimensions. The applications of these estimates in Section 3 are intrinsically one-dimensional, so those results do not extend to higher dimensions. Nevertheless, we believe these general estimates may be of use elsewhere.

We recall the abstract eigenfunction expansion dubbed BGK expansions in [43] after work of Berezinski, Browden, Garding, Gel'fand, and Kac (see [43] for references). In the discrete case, they take the following form. Let $V$ be an arbitrary function on $\mathbb{Z}^{\nu}$. Let $(H u)(n)=\sum_{|\ell|=1} u(n+\ell)+V(n) u(n)$. Then there exist measures $\left\{d \rho_{k}(E)\right\}_{k=1}^{\infty}$ on $\mathbb{R}$ and a measure $d \rho_{\infty}(E)$ so that the $d \rho$ 's are mutually singular [1,13,45]. Moreover, for a.e. $E$ w.r.t. $d \rho_{k}(E)$, there exist $k$ linearly independent functions $\left\{u_{j, k}(n ; E)\right\}_{j=1}^{k}$ on $\mathbb{Z}^{\nu}$ so that

(i) $\sum_{|\ell|=1} u_{j}(n+\ell)+(V(n)-E) u_{j}(n)=0$. 
(ii) For any $f$ on $\mathbb{Z}^{\nu}$ of finite support, define $a_{j, k}(f)(E)=\sum_{n} \overline{u_{j, k}(n ; E)} f(n)$. Then

$$
a_{j, k}(H f)(E)=E a_{j, k}(f)(E)
$$

and for any $f, g$ of finite support,

$$
\langle f, g\rangle=\sum_{k=1}^{" \infty} \sum_{j=1}^{k} \int \overline{a_{j, k}(f)(E)} a_{j, k}(g)(E) d \rho_{k}(E)
$$

with an explicit $\rho_{\infty}$-term intended in $\sum_{k=1}$ ".

Pick $f=g=\delta_{n}$, a Kronecker delta function at $n$. Then (A.1) becomes

$$
\sum_{k=1}^{\infty \infty} \sum_{j=1}^{k} \int\left|u_{j, k}(n ; E)\right|^{2} d \rho_{k}(E)=1 .
$$

This is essentially (2.6D) except in arbitrary dimension. In the one-dimensional case, $d \rho_{k}=0$ for $k \neq 1$. If we define $d \tilde{\rho}(E)=|u(1 ; E)|^{2} d \rho_{1}$ and $\tilde{u}(n ; E)=u(n ; E) u(1 ; E)^{-1}$, then (A.2) is exactly (2.6D).

In the continuum case, the situation is similar. One needs some minimal local regularity on $V$ (see [43]). Using the fact that $e^{-t H}(x, x)=0\left(t^{-1 / 2}\right)$ as $t \downarrow 0$, one can show that as $f \rightarrow \delta_{x}$, a $\delta$-function at $x,\left(f,(H+c)^{-\ell} f\right)$ stays finite and bounded in $x$ so long as $2 \ell>\nu$. Thus, (A.2) in the continuum case becomes

$$
\sum_{k=1}^{\prime \infty} \sum_{j=1}^{k} \int\left|u_{j, k}(x ; E)\right|^{2} \frac{d \rho_{k}(E)}{(1+|E|)^{\ell}} \leq C
$$

uniformly in $x$ where $\ell>\frac{\nu}{2}$.

As in Section 3, from these bounds and Fatou's lemma, we get bounds like before for a.e. $E$ w.r.t. $\sum_{k} d \rho_{k}(E)$,

$$
\varliminf_{L \rightarrow \infty} \frac{1}{(2 L+1)^{\nu}} \sum_{|n| \leq L}|u(n ; E)|^{2}<\infty .
$$

\section{REFERENCES}

1. N. Aronszajn, On a problem of Weyl in the theory of Sturm-Liouville equations, Am. J. Math. 79 (1957), 597-610.

2. J. Avron and B. Simon, Almost periodic Schrödinger operators, II. The integrated density of states, Duke Math. J. 50 (1983), 369-391. 
3. N. Brenner and S. Fishman, Pseudo-randomness and localization, Nonlinearity 4 (1992), 211-235.

4. R. Carmona, One-dimensional Schrödinger operators with random or deterministic potentials, New spectral types, J. Funct. Anal. 51 (1983), 229-258.

5. R. Carmona and J. Lacroix, Spectral Theory of Random Schrödinger Operators, Birkhäuser, Boston, 1990.

6. I.P. Cornfeld, S.V. Fomin, and Ya.G. Sinai, Ergodic Theory, Springer, New YorkHeidelberg-Berlin, 1982.

7. H.L. Cycon, R.G. Froese, W. Kirsch, and B. Simon, Schrödinger Operators, Springer, Berlin-Heidelberg-New York, 1987.

8. E.B. Davies and B. Simon, Scattering theory for systems with different spatial asymptotics on the left and right, Commun. Math. Phys. 63 (1978), 277-301.

9. E.B. Davies and B. Simon, $L^{1}$-properties of intrinsic Schrödinger semigroups, J. Funct. Anal. 65 (1986), 126-146.

10. P. Deift and B. Simon, Almost periodic Schroödinger operators, III. The absolutely continuous spectrum in one dimension, Commun. Math. Phys. 90 (1983), 389-411.

11. F. Delyon, Apparition of purely singular continuous spectrum in a class of random Schrödinger operators, J. Statist. Phys. 40 (1985), 621-630.

12. F. Delyon, B. Simon, and B. Souillard, From power pure point to continuous spectrum in disordered systems, Ann. Inst. H. Poincaré 42 (1985), 283-309.

13. W. Donoghue, On the perturbation of the spectra, Commun. Pure Appl. Math. 18 (1965), 559-579.

14. H. Furstenberg, Strict ergodicity and transformations of the torus, Am. J. Math. 83 (1961), 573-601.

15. D.J. Gilbert and D. Pearson, On subordinacy and analysis of the spectrum of onedimensional Schrödinger operators, J. Math. Anal. 128 (1987), 30-56.

16. I. Goldsheid and E. Sorets, Lyapunov exponents of the Schrödinger equation with certain classes of ergodic potentials, Amer. Math. Soc. Trans. (2) 171 (1996), 73-80.

17. A.Ya. Gordon, Deterministic potential with a pure point spectrum, Math. Notes $\mathbf{4 8}$ (1990), 1197-1203.

18. M. Griniasty and S. Fishman, Localization by pseudorandom potentials in one dimension, Phys. Rev. Lett. 60 (1988), 1334-1337. 
19. J.P. Guillement, B. Helffer, and P. Treton, Walk inside Hofstadter's butterfly, J. Phys. France 50 (1989), 2019-2058.

20. B. Helffer and J. Sjöstrand, Semi-classical analysis for Harper's equation, III. Cantor structure of the spectrum, Mém. Soc. Math. France (N.S.) 39 (1989), 1-139.

21. K. Ishii, Localization of eigenstates and transport phenomena in one-dimensional disordered systems, Suppl. Prog. Theor. Phys. 53 (1973), 77-138.

22. S. Jitomirskaya, to be published.

23. S. Jitomirskaya and Y. Last, Dimensional Hausdorff properties of singular continuous spectra, Phys. Rev. Lett. 76 (1996), 1765-1769.

24. S. Jitomirskaya and Y. Last, Power law subordinacy and singular spectra, I. Half-line operators, in preparation.

25. S. Jitomirskaya and B. Simon, Operators with singular continuous spectrum, III. Almost periodic Schrödinger operators, Commun. Math. Phys. 165 (1994), 201-205.

26. S. Kahn and D.B. Pearson, Subordinacy and spectral theory for infinite matrices, Helv. Phys. Acta 65 (1992), 505-527.

27. Y. Katznelson, An Introduction to Harmonic Analysis, Dover, New York, 1976.

28. A.A. Kiselev, Absolutely continuous spectrum of one-dimensional Schrödinger operators and Jacobi matrices with slowly decreasing potentials, Commun. Math. Phys. 179 (1996), 377-400.

29. A. Kiselev, Y. Last, and B. Simon, Modifed Prüfer and EFGP transforms and the spectral analysis of one-dimensional Schrödinger operators, to appear in Commun. Math. Phys.

30. S. Kotani, Ljapunov indices determine absolutely continuous spectra of stationary random one-dimensional Schrödinger operators, Stochastic Analysis (K. Ito, ed.), pp. 225-248, North Holland, Amsterdam, 1984.

31. S. Kotani, Support theorems for random Schrödinger operators, Commun. Math. Phys. 97 (1985), 443-452.

32. S. Kotani, Jacobi matrices with random potential taking finitely many values, Rev. Math. Phys. 1 (1989), 129-133.

33. Y. Last, Zero measure spectrum for the almost Mathieu operator, Commun. Math. Phys. 164 (1994), 421-432.

34. Y. Last, Conductance and Spectral Properties, D.Sc. thesis, Technion, 1994. 
35. Y. Last, Periodic approximants of Jacobi matrices, II. Complete determination of the absolutely continuous spectrum, in preparation.

36. Y. Last and B. Simon, Modified Prüfer and EFGP transforms and deterministic models with dense point spectrum, to appear in J. Funct. Anal.

37. B.M. Levitan and I.S. Sargsjan Introduction to Spectral Theory, Trans. Math. Monographs, 39, Amer. Math. Soc., Providence, RI, 1976.

38. L. Pastur, Spectral properties of disordered systems in the one-body approximation, Commun. Math. Phys. 75 (1980), 167-196.

39. D. Pearson, Singular continuous measures in scattering theory, Commun. Math. Phys. 60 (1978), 13-36.

40. D. Pearson, Pathological spectral properties, Mathematical Problems in Theoretical Physics, pp. 49-51, Lecture Notes in Physics No. 80, Springer, Berlin-New York, 1979.

41. D. Ruelle, Ergodic theory of differentiable dynamical systems, Publ. Math. IHES 50 (1979), 275-306.

42. I. Sch'nol, On the behavior of the Schrödinger equation, Mat. Sb. 42 (1957), 273-286 [in Russian].

43. B. Simon, Schrödinger semigroups, Bull. Amer. Math. Soc. 7 (1982), 447-526.

44. B. Simon, Some Jacobi matrices with decaying potentials and dense point spectrum, Commun. Math. Phys. 87 (1982), 253-258.

45. B. Simon, Spectral analysis and rank one perturbations and applications, CRM Lecture Notes Vol. 8 (J. Feldman, R. Froese, L. Rosen, eds.), pp. 109-149, Amer. Math. Soc., Providence, RI, 1995.

46. B. Simon and T. Spencer, Trace class perturbations and the absence of absolutely continuous spectrum, Commun. Math. Phys. 125 (189), 113-126.

47. B. Simon and G. Stolz, Operators with singular continuous spectrum, V. Sparse potentials, Proc. Amer. Math. Soc. 124 (1996), 2073-2080.

48. D.J. Thouless, Localization by a potential with slowly varying period, Phys. Rev. Lett. 61 (1988), 2141-2143. 